QUARTERLY OF APPLIED MATHEMATICS

VOLUME LXIII, NUMBER 2

JUNE 2005, PAGES 225-249

S $0033-569 X(05) 00939-6$

Article electronically published on April 12, 2005

\title{
WAVE PATTERNS FOR SHALLOW WATER EQUATIONS
}

\author{
BY \\ CHIU-YA LAN (Institute of Mathematics, Academia Sinica, Nankong, Taipei 11529, Taiwan) \\ AND \\ HUEY-ER LIN (Institute of Mathematics, Academia Sinica, Nankong, Taipei 11529, Taiwan)
}

Abstract. We consider the time-asymptotic behavior of the system of shallow water equations with one bump in one dimension. Our main interest is in the issue of nonlinear stability and instability of the waves, particularly for the transonic flow. In this paper, the formation of the asymptotic wave patterns is done by combining elementary nonlinear waves, shock and rarefaction waves for the conservation laws, and stationary waves. We also describe the bifurcations of the wave patterns as the end states vary.

1. Introduction. Consider the system of shallow water (Saint-Venant) equations:

$$
\begin{aligned}
h_{t}+(h u)_{x} & =0, \\
(h u)_{t}+\left(h u^{2}+\frac{1}{2} g h^{2}\right)_{x} & =-g h B_{x},
\end{aligned}
$$

where $B(x)$ represents the bottom elevation, $h$ is the fluid depth above the bottom, $u$ denotes the velocity, and $g$ is the gravitational constant.

There is renewed interest in the shallow water equations from the geophysical applications; see [1], [2], [3], [4] and references therein. Our main interest is in the issue of nonlinear stability and instability of the waves, particularly for the transonic flows. This issue can be studied by considering the time asymptotic behavior of the system (1.1)(1.2) for given upstream state $U_{l}=\left(h_{l}, u_{l}\right)$ and downstream state $U_{r}=\left(h_{r}, u_{r}\right)$. From a physical perspective, the fluid speed $u$ and the fluid depth $h$ are both assumed to be positive. For simplicity we assume that the bottom is flat outside a finite region:

$$
B(x)=0 \text {, for } x \in(-\infty,-1) \cup(1, \infty) .
$$

There are complex nonlinear wave interactions in a general flow. However, time-asymptotically, these interactions will decay and the flow will tend to an asymptotic wave pattern consisting of three noninteracting waves: a nonpositive speed wave for $x \in$ $(-\infty,-1)$, a stationary wave for $x \in[-1,1]$ and a nonnegative speed wave for $x \in(1, \infty)$.

Received April, 2003.

2000 Mathematics Subject Classification. Primary 76B15, 76H05, 35L65.

E-mail address: cylan@math.nsysu.edu.tw

E-mail address: helin@math.ntnu.edu.tw

(C)2005 Brown University 
In other words, these waves are governed by either the conservation laws or the stationary equations, respectively:

$$
\begin{aligned}
h_{t}+(h u)_{x} & =0, \\
(h u)_{t}+\left(h u^{2}+\frac{1}{2} g h^{2}\right)_{x} & =0,
\end{aligned}
$$

or

$$
\begin{aligned}
(h u)_{x} & =0, \\
\left(h u^{2}+\frac{1}{2} g h^{2}\right)_{x} & =-g h B_{x} .
\end{aligned}
$$

In Sec. 2, we consider these elementary nonlinear waves, shock and rarefaction waves for the conservation laws, and stationary waves. The combination of these waves to form the asymptotic wave patterns is done in Sec. 3, which is the main part of the present paper. We carry out the complete analysis for the case when the bump $B(x)$ is not too large, consistent with the validity of the model, and in the interesting situation when the flow is around transonic. Assuming that the flow is to the right, $u>0$, it is transonic if the characteristic speed

$$
\lambda_{1}(h, u)=u-\left.\sqrt{g h}\right|_{\left(h, u=C_{0} / h\right)}
$$

of the system changes signs. In this case, there is the coupling of shock and rarefaction waves with the stationary waves which yield rich nonlinear behavior. In Sec. 4 we summarize the results in the previous section and study the bifurcations of the wave patterns as the end states vary. Of particular interest is the bifurcation phenomena as the supersonic upstream state varies around the critical state corresponding to the sonic state at the center of the bump. The number of solutions change from one to two to three in some range with the number of instability solutions remaining constant; c.f. Figures 16 to 19. There is the appearance of a semistable situation. The results differ qualitatively from the other systems of hyperbolic conservation laws with a source; see [7] and references therein. There is the study of general systems of conservation laws with a source; see [6]. Our results show that there are new and striking phenomena for the shallow water equations. The equations may be viewed as the most basic model of conservation laws with a source; thus, the results obtained here should be indicative for other systems.

2. Nonlinear Waves. For simplicity, we assume that $B$ is smooth, symmetric, and consists of one bump at $x=0$ with height $r ; B$ vanishes outside $|x|<1$.

We first study the region $x \notin[-1,1]$, where the system $(1.1)(1.2)$ is reduced to a system of hyperbolic conservation laws:

$$
\begin{aligned}
h_{t}+(h u)_{x} & =0, \\
(h u)_{t}+\left(h u^{2}+\frac{1}{2} g h^{2}\right)_{x} & =0 .
\end{aligned}
$$

The characteristic speeds are $\lambda_{1}(h, u)=u-\sqrt{g h}, \lambda_{2}(h, u)=u+\sqrt{g h}$ and so $\lambda_{2}$ is always positive for $h>0, u>0$. Given a fixed $\hat{U}=(\hat{h}, \hat{u})$ there are two rarefaction waves: a 
1-rarefaction wave $R_{1}$ and a 2 -rarefaction wave $R_{2}$ :

$$
\begin{aligned}
& R_{1}: u=\hat{u}-2(\sqrt{g h}-\sqrt{g \hat{h}}), 0<h<\hat{h}, \\
& R_{2}: u=\hat{u}+2(\sqrt{g h}-\sqrt{g \hat{h}}), \hat{h}<h .
\end{aligned}
$$

A discontinuity wave $\left(h_{-}, u_{-} ; h_{+}, u_{+}\right)$for $(1.3)(1.4)$ with speed $s$ satisfies the RankineHugoniot condition

$$
\begin{aligned}
s\left(h_{+}-h_{-}\right) & =\left(h_{+} u_{+}-h_{-} u_{-}\right), \\
s\left(h_{+} u_{+}-h_{-} u_{-}\right) & =\left(h_{+} u_{+}^{2}+\frac{1}{2} g h_{+}^{2}-h_{-} u_{-}^{2}-\frac{1}{2} g h_{-}^{2}\right) .
\end{aligned}
$$

For the fixed state $\hat{U}=(\hat{h}, \hat{u})$, the Hugoniot set

$$
\begin{aligned}
H(\hat{h}, \hat{u}) \equiv\left\{\sigma\left(\begin{array}{c}
h-\hat{h} \\
h u-\hat{h} \hat{u}
\end{array}\right)\right. & =\left(\begin{array}{l}
h u-\hat{h} \hat{u} \\
h u^{2}+\frac{1}{2} g h^{2}-\hat{h} \hat{u}^{2}-\frac{1}{2} g \hat{h}^{2}
\end{array}\right) \\
\text { for some scalar } \sigma & =\sigma(\hat{h}, \hat{u} ; h, u)\}
\end{aligned}
$$

consists of two curves $S_{1}(\hat{h}, \hat{u}), S_{2}(\hat{h}, \hat{u})$ :

$$
\begin{aligned}
& S_{1}: \quad u=\hat{u}-\sqrt{\frac{g(h+\hat{h})}{2 h \hat{h}}}(h-\hat{h}), \\
& S_{2}: \quad u=\hat{u}+\sqrt{\frac{g(h+\hat{h})}{2 h \hat{h}}}(h-\hat{h}) .
\end{aligned}
$$

The Rankine-Hugoniot condition (2.1)(2.2) is equivalent to

$$
s=\sigma\left(h_{-}, u_{-} ; h_{+}, u_{+}\right), \quad\left(h_{+}, u_{+}\right) \in S_{i}\left(h_{-}, u_{-}\right), i=1 \text { or } 2 .
$$

A shock wave for the system (1.3)(1.4) is required to satisfy the Lax admissibility criterion $[5]$ :

$$
\begin{aligned}
& S_{1}: \quad u=\hat{u}-\sqrt{\frac{g(h+\hat{h})}{2 h \hat{h}}}(h-\hat{h}), \quad h>\hat{h}, \\
& S_{2}: \quad u=\hat{u}+\sqrt{\frac{g(h+\hat{h})}{2 h \hat{h}}}(h-\hat{h}), \quad h<\hat{h} .
\end{aligned}
$$

In addition to the above elementary waves for conservation laws, in the region $x \in[-1,1]$, there are stationary waves satisfying differential equations

$$
\begin{aligned}
(h u)_{x} & =0, \\
\left(h u^{2}+\frac{1}{2} g h^{2}\right)_{x} & =-g h B_{x}
\end{aligned}
$$

or, equivalently,

$$
\begin{aligned}
& \qquad \begin{array}{l}
h u=C_{0} \\
f(h)_{x}
\end{array}=-g h B_{x} \\
& \text { where } \quad f(h) \equiv \frac{C_{0}^{2}}{h}+\frac{1}{2} g h^{2} .
\end{aligned}
$$


Note that $f(h)$ is a convex function of $h$ for $h>0$. The minimum of $f(h), h>0$, is attained at the sonic state

$$
h_{0}=\left(\frac{C_{0}^{2}}{g}\right)^{1 / 3} \quad \text { (see Fig. 1). }
$$

It's noticed that for the stationary solutions,

$$
\begin{gathered}
\lambda_{1}(h, u)=u-\left.\sqrt{g h}\right|_{\left(h, u=C_{0} / h\right)}=\frac{C_{0}}{h}-\sqrt{g h} \begin{cases}>0 & \text { for } h<h_{0}, \\
=0 & \text { for } h=h_{0}, \\
<0 & \text { for } h>h_{0},\end{cases} \\
f^{\prime}(h)=g h-\frac{C_{0}^{2}}{h^{2}}=-\left.\lambda_{1} \lambda_{2}\right|_{\left(h, u=C_{0} / h\right) .}
\end{gathered}
$$

Let $\bar{h}$ be the initial value at $x=-1$ of the stationary equation

$$
\left\{\begin{array}{l}
f(h)_{x}=-g h B_{x} \\
h(-1)=\bar{h}
\end{array}\right.
$$

such that $h(x)$ is continuous in $-1 \leq x \leq 1$ with $h\left(0^{-}\right)=h_{0}$. By solving the initial value problem above, it is easy to see that $\bar{h}$ satisfies the cubic equation

$$
H\left(h ; C_{0}\right) \equiv h^{3}-\left(r+\frac{3}{2} g^{\frac{-1}{3}} C_{0}^{\frac{2}{3}}\right) h^{2}+\frac{C_{0}^{2}}{2 g}=0 .
$$

Lemma 2.1. For the cubic equation (2.6) with fixed $C_{0}$, there are exactly two positive roots; one is greater than $h_{0}$ and the other less than $h_{0}$.

Proof. Since the derivative of $H(h)$ is

$$
H^{\prime}(h)=3 h^{2}-2\left(r+\frac{3}{2} g^{\frac{-1}{3}} C_{0}^{\frac{2}{3}}\right) h,
$$

$H^{\prime}(h)=0$ if and only if $h=0$ or $h=\frac{2}{3}\left(r+\frac{3}{2} g^{\frac{-1}{3}} C_{0}^{\frac{2}{3}}\right)$. By straightforward calculations, we have

$$
\begin{aligned}
& \lim _{h \rightarrow-\infty} H(h)=-\infty, \\
& H(0)=\frac{C_{0}^{2}}{2 g}>0, \quad H\left(\frac{2}{3}\left(r+\frac{3}{2} g^{\frac{-1}{3}} C_{0}^{\frac{2}{3}}\right)\right)<0, \\
& \lim _{h \rightarrow \infty} H(h)=\infty .
\end{aligned}
$$

These imply that $H(h)=0$ has three real roots; one is negative and the other two are positive. Furthermore, from $H\left(h_{0}\right)<0$, one of the positive zeros is less than $h_{0}$ and the other is greater than $h_{0}$.

Denote the two positive zeros of (2.6) by $h_{c}$ and $h_{*}, h_{c}<h_{*}$. By differential inequality, if the initial value $\bar{h}$ of (2.5) satisfies $0<\bar{h} \leq h_{c}$ or $\bar{h} \geq h_{*}$, the initial value problem (2.5) has a continuous solution. It should be mentioned that $h_{c}$ and $h_{*}$ are dependent on the constant $C_{0}$, which can be regarded as a parameter of the stationary equation. The following lemma is concerned with the dependence of $h_{*}$ on the parameter $C_{0}$.

Lemma 2.2. Let $C_{0 i}, i=1,2$, be two values, $0<C_{01}<C_{02}$; then the solutions of (2.3)(2.4) $h=h_{i}$ with $C_{0}=C_{0 i}, i=1,2$, have the monotonicity property that $h_{1 *}<h_{2 *}$. 


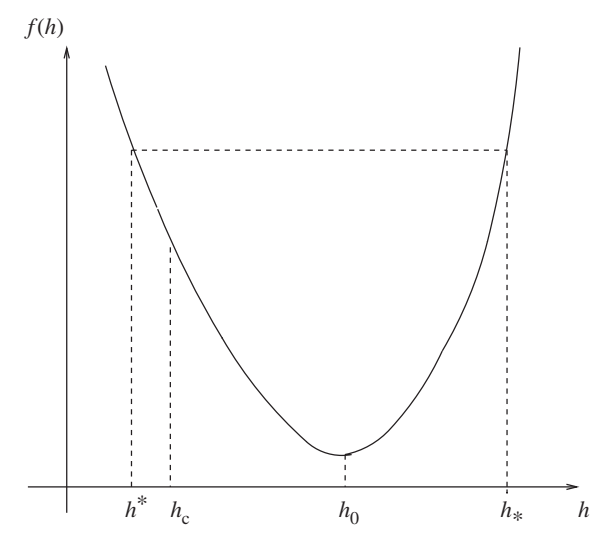

FIG. 1.

Proof. Recall that $h_{i 0} \equiv\left(C_{0 i}^{2} / g\right)^{\frac{1}{3}}$ and $h_{i *}\left(>h_{i 0}\right)$ are the initial values of the stationary equations at $x=-1$ such that the solutions, say $h_{i}(x)$, attain $h_{i 0}$ at $x=0^{-}$. Let $\bar{h}(x)$ be the solution of

$$
\left\{\begin{array}{l}
\left(f_{1}(h)\right)_{x}=-g h B_{x}(x) \\
h(-1)=h_{2 *}
\end{array}\right.
$$

From $f_{i}^{\prime}(h)=\frac{-C_{0 i}^{2}}{h^{2}}+g h$,

$$
f_{1}^{\prime}(h)>f_{2}^{\prime}(h)>0 \text { for } h>h_{20}
$$

and so

$$
\frac{-g h B_{x}(x)}{f_{2}^{\prime}(h)}<\frac{-g h B_{x}(x)}{f_{1}^{\prime}(h)}<0 \quad \text { for }-1 \leq x \leq 0, h>h_{20} .
$$

Then by differential inequality, we get

$$
\left(h_{10}<h_{20}=\right) h_{2}\left(0^{-}\right)<\bar{h}(0) .
$$

Furthermore, since $h_{1}(x)$ and $\bar{h}(x)$ satisfy the same differential equation and $h_{1}\left(0^{-}\right)=$ $h_{10}<\bar{h}(0)$ it implies $h_{1 *} \equiv h_{1}(-1)<\bar{h}(-1)=h_{2 *}$.

Key Lemma 2.3. Let $0<h_{1}<h_{2} \leq h_{0} \leq h_{3}<h_{4}\left(0<h_{2}<h_{1} \leq h_{0} \leq h_{4}<h_{3}\right)$ be the points such that $f\left(h_{1}\right)=f\left(h_{4}\right)$ and $f\left(h_{2}\right)=f\left(h_{3}\right)$. Assume that both $h^{(1)}$ and $h^{(2)}$ satisfy the stationary equation $(2.3)(2.4) ; h^{(1)}(x)$ achieves $h_{1}$ at $x_{1}$ and $h_{2}$ at $x_{2}, h^{(2)}(x)$ achieves $h_{4}$ at $x_{1}$ and $h_{3}$ at $\bar{x}_{2}$, where $-1 \leq x_{1}<x_{2}, \bar{x}_{2} \leq 0\left(0 \leq x_{1}<x_{2}, \bar{x}_{2} \leq 1\right)$ (see Fig. 2). Then we have $x_{2}>\bar{x}_{2}$; it means the path $x_{2}-x_{1}$ of the stationary wave connecting $h_{1}$ to $h_{2}\left(h_{2}\right.$ to $\left.h_{1}\right)$ is longer than the path $\bar{x}_{2}-x_{1}$ of that connecting $h_{4}$ to $h_{3}\left(h_{3}\right.$ to $\left.h_{4}\right)$. 
Proof. Since $h^{(1)}(x)$ and $h^{(2)}(x)$ are solutions of the stationary equation,

$$
\begin{aligned}
(\triangle) \quad f\left(h_{2}\right)-f\left(h_{1}\right) & =\int_{x_{1}}^{x_{2}}-g h^{(1)}(s) B_{x}(s) d s, \\
(\triangle \triangle) & f\left(h_{3}\right)-f\left(h_{4}\right)=\int_{x_{1}}^{\bar{x}_{2}}-g h^{(2)}(s) B_{x}(s) d s .
\end{aligned}
$$

By the assumptions, the values of the left-hand sides in $(\triangle)$ and $(\triangle \triangle)$ are equal. Then

$$
\begin{aligned}
& \int_{x_{1}}^{x_{2}}-g h^{(1)}(s) B_{x}(s) d s=\int_{x_{1}}^{\bar{x}_{2}}-g h^{(2)}(s) B_{x}(s) d s \\
\Rightarrow & \int_{x_{1}}^{x_{2}}-g\left(h^{(2)}(s)-h^{(1)}(s)\right) B_{x}(s) d s+\int_{x_{2}}^{\bar{x}_{2}}-g h^{(2)}(s) B_{x}(s) d s=0 .
\end{aligned}
$$

The first term in the left-hand side is negative (positive). Due to $-g h^{(2)}(s) B_{x}(s)<0(>0)$ for all $-1 \leq s \leq 0(0 \leq s \leq 1)$, we have $x_{2}>\bar{x}_{2}$.

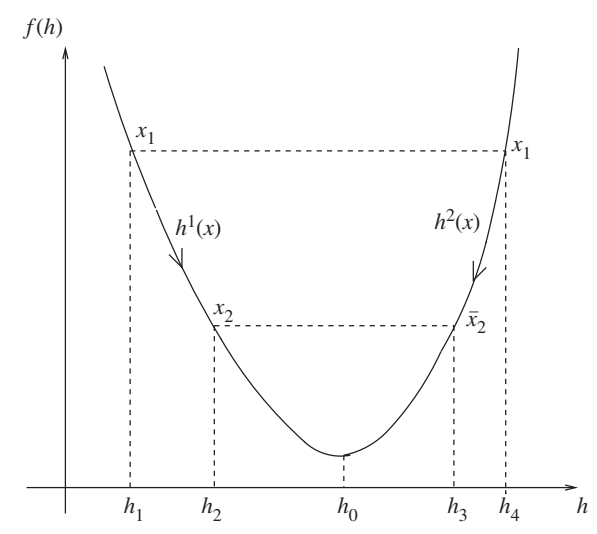

FIG. 2.

Let $h^{*}>0$ be the point such that $f\left(h^{*}\right)=f\left(h_{*}\right)$. According to the definitions of $h_{*}, h_{c}, h^{*}$ and using the key lemma, it immediately follows that $0<h^{*}<h_{c}<h_{0}<h_{*}$ (see Fig. 1).

3. Time-asymptotic behavior. Given an upstream state $U_{l}=\left(h_{l}, u_{l}\right), C_{0} \equiv h_{l} u_{l}$, we may define $h^{*}\left(C_{0}\right), h_{c}\left(C_{0}\right), h_{0}\left(C_{0}\right), h_{*}\left(C_{0}\right)$ according to Lemma 2.1. To study different time-asymptotic behaviors, we divide the domain of $h$ into several regions:

$$
\begin{aligned}
I & =\left\{U_{l} \mid 0<h_{l} \leq h^{*}, h_{l} u_{l}=C_{0}\right\}, \\
I I & =\left\{U_{l} \mid h^{*}<h_{l} \leq h_{c}, h_{l} u_{l}=C_{0}\right\}, \\
I I I & =\left\{U_{l} \mid h_{c}<h_{l}<h_{0}, h_{l} u_{l}=C_{0}\right\}, \\
I V & =\left\{U_{l} \mid h_{0}<h_{l} \leq h_{*}, h_{l} u_{l}=C_{0}\right\}, \\
V & =\left\{U_{l} \mid h_{*}<h_{l}, h_{l} u_{l}=C_{0}\right\} .
\end{aligned}
$$

First, we have the following observations: for $U_{l}=\left(h_{l}, u_{l}\right) \in I, I I, I I I$ or $I V$, since the speed of the asymptotic wave in $x<-1$ should be nonpositive, the flow is a constant 
state or a 1-shock $\left(U_{l} ; U_{-1}\right)$ with negative speed in $x<-1$; for $U_{l} \in V$, the flow is a constant state or a 1 -wave $\left(U_{l} ; U_{-1}\right)$ with negative speed in $x<-1$. To study the behavior in more detail, we consider the following cases:

Case 3.1: $U_{l} \in I$ and $0<h_{l}<h^{*}$. We have the following possible asymptotic wave patterns:

Subcase (i)

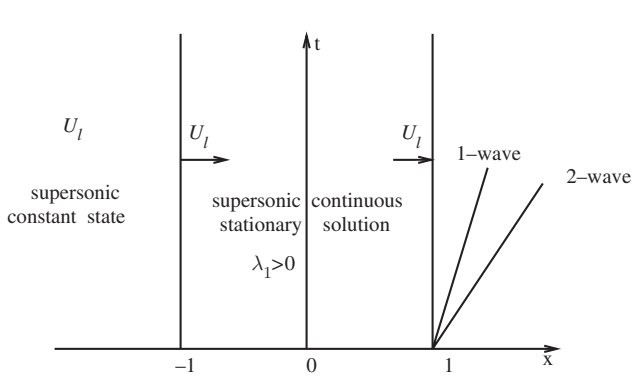

FIG. 3-1

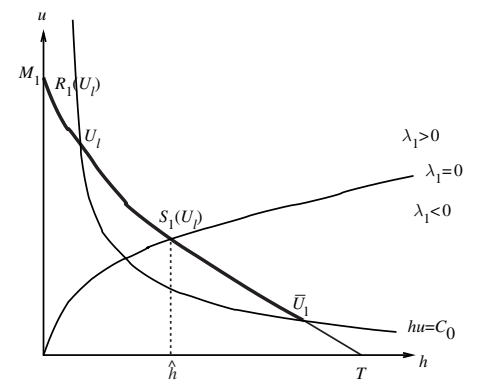

Fig. 3-2 The bold curves represent all the possible states $\bar{U}$ which are connected to $U_{r}$ by 2 -waves in $x>1$.

The wave pattern consists of a supersonic constant state $U_{l}=\left(h_{l}, u_{l}\right)$ in $x \leq-1$, a stationary wave in $-1 \leq x \leq 1$, and either a 1 -wave with positive speed followed by a 2 -wave or a 2 -wave in the region $x \geq 1$. The stationary wave is supersonic and continuous, and attains the state $U_{l}$ at $x=1^{-}$as the result of the symmetry of the bump. The 1-wave, denoted by $\left(U_{l} ; \bar{U}\right)$, is either a supersonic 1-shock if $h_{l}<\bar{h}<\hat{h},\left(\bar{U}_{l}=\left(\bar{h}_{l}, \bar{u}_{l}\right) \in S_{1}\left(U_{l}\right)\right.$ with the shock speed $s\left(U_{l}, \bar{U}_{l}\right)=0 ; \quad \hat{U}=(\hat{h}, \hat{u}) \in S_{1}\left(U_{l}\right)$ with $\left.\lambda_{1}(\hat{U})=0\right)$, a transonic 1-shock if $\hat{h} \leq \bar{h}<\bar{h}_{l}$, or a supersonic 1-rarefaction if $0<\bar{h}<h_{l}$. The 2 -wave is a 2 -rarefaction wave or a 2 -shock wave.

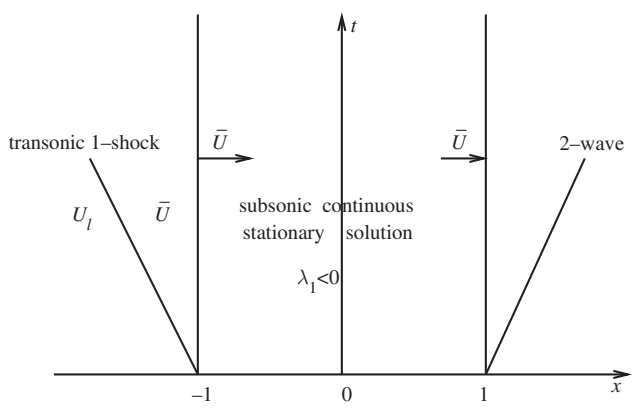

FIG. 4-1

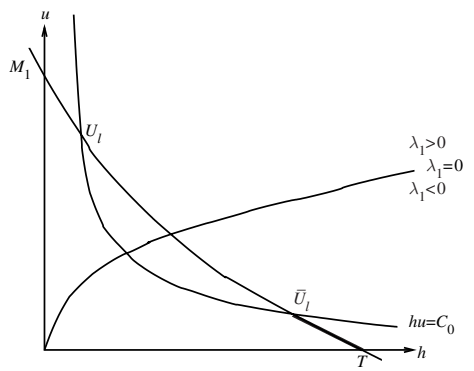

FIG. 4-2 
Subcase (ii) The wave pattern consists of a 1-shock in $x<-1$, a stationary wave in $-1 \leq x \leq 1$, and a 2 -wave in $x>1$. The 1 -shock is transonic, denoted by $\left(U_{l} ; \bar{U}\right)\left(\bar{U}=(\bar{h}, \bar{u}) \in S_{1}\left(U_{l}\right)\right.$ with $\left.\bar{h}>\bar{h}_{l}\right)$. For $C_{1} \equiv \bar{h} \bar{u}<\bar{h}_{l} \bar{u}_{l}=C_{0}$, the stationary wave, denoted by $\left(h(x), u(x)=\frac{C_{1}}{h(x)}\right)$, is continuous, subsonic by Lemma 2.2, and attains $\bar{U}$ at $x=1^{-}$. The 2 -wave is a 2-rarefaction wave or a 2 -shock wave.

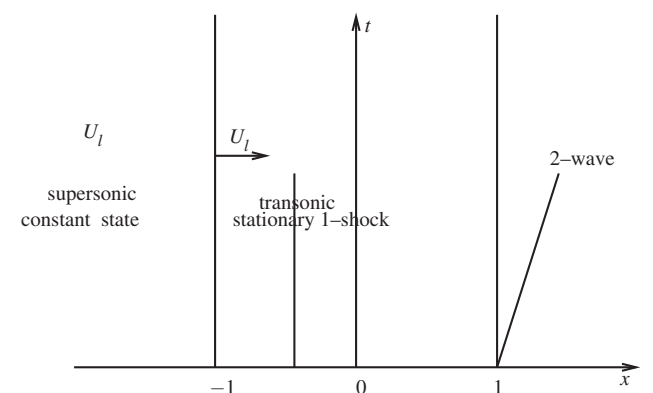

FIG. 5-1

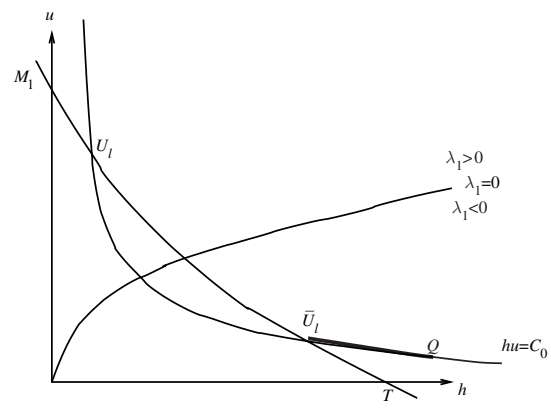

FIG. 5-2

Subcase (iii) The wave pattern consists of a supersonic constant state $U_{l}$ in $x<-1$, a stationary wave with 1 -shock in $-1 \leq x \leq 1$, and a 2 -wave in $x>1$. The 1 -shock above the bump $(-1 \leq x \leq 1)$ is stationary and transonic. For $0<h_{l} \leq h^{*}$, the 1-shock can occur at any position, say $x_{s}$, above the bump by Key Lemma 2.3. However, the depth $h\left(1^{-}\right)$the stationary wave attains at $x=1^{-}$is dependent on the position $x_{s}$ of the 1-shock. The dependence is characterized by the following lemmas.

Lemma 3.1. The stationary wave with 1 -shock at $x_{s},-1 \leq x_{s} \leq 0$, attains the same state at $x=1$ as the stationary wave with 1 -shock at $-x_{s}$.

Proof. Let $h_{1}(x)$ and $h_{2}(x)$ be the depth functions of the stationary waves satisfying (2.3)(2.4) with the same initial value at $x=-1$ and transonic stationary 1-shocks at $x_{s}$ and $-x_{s}$, respectively. Then

$$
h_{1}\left(x_{s}^{-}\right)=h_{2}\left(x_{s}\right) .
$$

Due to the symmetry of the bump, we also have

$$
h_{2}\left(\left(-x_{s}\right)^{-}\right)=h_{2}\left(x_{s}\right) .
$$

From the equalities above, we obtain

$$
h_{1}\left(x_{s}^{-}\right)=h_{2}\left(\left(-x_{s}\right)^{-}\right) .
$$

Since the shock speeds are both zeros, the Rankine-Hugoniot condition (2.1)(2.2) implies

$$
\begin{aligned}
& f\left(h_{1}\left(x_{s}^{-}\right)\right)=f\left(h_{1}\left(x_{s}^{+}\right)\right), \\
& f\left(h_{2}\left(\left(-x_{s}\right)^{-}\right)\right)=f\left(h_{2}\left(\left(-x_{s}\right)^{+}\right)\right) .
\end{aligned}
$$


Using the convexity of $f(h)$ for $h>0$, we get

$$
h_{1}\left(x_{s}^{+}\right)=h_{2}\left(\left(-x_{s}\right)^{+}\right) .
$$

Due to the symmetry of the bump, we have

$$
h_{1}\left(x_{s}^{+}\right)=h_{1}\left(\left(-x_{s}\right)\right)
$$

So

$$
h_{1}\left(\left(-x_{s}\right)\right)=h_{2}\left(\left(-x_{s}\right)^{+}\right)>h_{0} .
$$

Since both $h_{1}(x)$ and $h_{2}(x)$ satisfy the same differential equations $(2.3)(2.4)$ in $0<$ $\left(-x_{s}\right)<x<1$ and have the same initial value at $x=\left(-x_{s}\right)^{+}$, it implies $h_{1}(1)=h_{2}(1)$ and hence $u_{1}(1)=u_{2}(1)$.

LEMma 3.2. Let $h_{i}(x), i=1,2$ be the depth functions of the stationary waves satisfying (2.3) (2.4) with the same initial value at $x=-1$ and stationary 1 - shock at $x_{s i}, i=1,2$, respectively. Assume $-1 \leq x_{s 1}<x_{s 2} \leq 0$; then $h_{1}(1)<h_{2}(1)$.

Proof. By Key Lemma 2.3, we know that the path of $h_{2}(x)$ from $h_{2}\left(x_{s 1}\right)$ to $h_{2}\left(x_{s 2}^{-}\right)$ is longer than the path of $h_{1}(x)$ from $h_{1}\left(x_{s 1}^{+}\right)$to $h_{2}\left(x_{s 2}^{+}\right)$(see Fig. 6). Hence $h_{1}$ attains the value $h_{2}\left(x_{s 2}^{+}\right)$at $y$ with $x_{s 1}<y<x_{s 2}<0$. It implies $h_{1}(0)<h_{2}(0)$ and hence $h_{1}(1)<h_{2}(1)$.

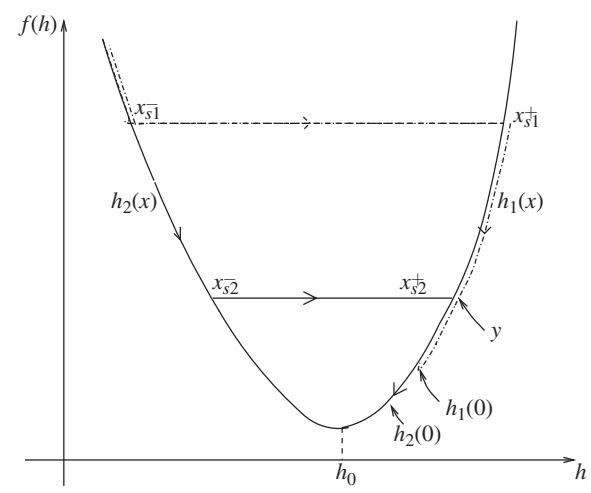

FIG. 6.

From Lemma 3.1 and Lemma 3.2, we also know that if the stationary 1- shocks occur at $x_{s 1}$ and $x_{s 2}$ with $0 \leq x_{s 1}<x_{s 2} \leq 1$, respectively, then $h_{1}(1)>h_{2}(1)$.

Case 3.2: $\quad U_{l} \in I$ and $h_{l}=h^{*}$.

There are richer wave patterns in this case than in Case 3.1. When the stationary 1-shock occurs at $x_{s}=-1$, a choke happens, which means that the stationary wave attains sonic state $U_{0}=\left(h_{0}, u_{0}\right)$ at the top of the bump $x=0$; the choke results in richer wave patterns. Besides all wave patterns described in Case 3.1, there are more possible asymptotic wave patterns as follows: 


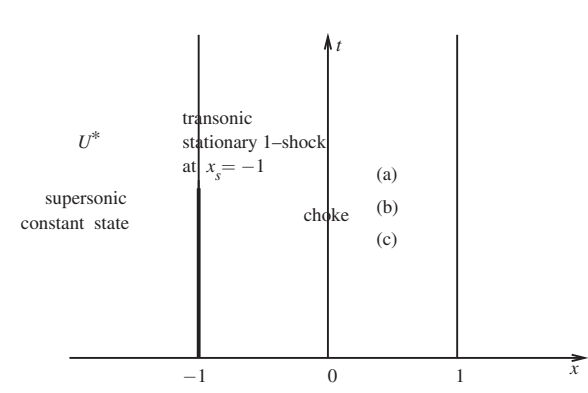

FIG. 7-1

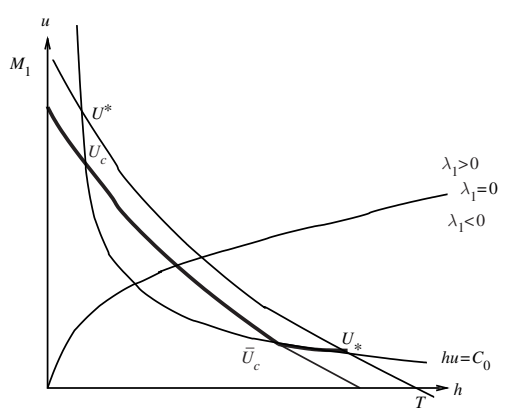

FIG. 7-2

Subcase (a) The wave pattern consists of a supersonic constant state $U^{*}$ in $x<-1$, a stationary wave with 1 -shock, denoted by $\left(U^{*} ; U_{*}\right)$, at $x_{s}=-1$ in the region $-1 \leq x \leq 1$ and a 2 -wave in $x>1$. The stationary wave is transonic in $-1 \leq x \leq 0$, attaining the sonic state $U_{0}$ at $x=0$ and continuous, subsonic in $0<x \leq 1$, attaining the state $U_{*}$ at $x=1$. The 2 -wave, denoted by $\left(U_{*} ; U_{r}\right)$, is a 2 -shock wave or a 2 -rarefaction wave.

Subcase (b) The wave pattern consists of a supersonic constant state $U^{*}$ in $x<-1$, a stationary wave with 1 -shock, denoted by $\left(U^{*} ; U_{*}\right)$, at $x_{s}=-1$ in the region $-1 \leq x \leq 1$ and either a 1 -wave followed by a 2 -wave in $x>1$ or a 2 -wave. The stationary wave is transonic in $-1 \leq x \leq 0$, attaining the sonic state $U_{0}$ at $x=0$ and continuous, supersonic in $0<x \leq 1$, attaining the state $U_{c}$ at $x=1$. The 1 -wave, denoted by $\left(U_{c} ; \bar{U}\right)$, is either a supersonic 1 -shock wave if $h_{c}<\bar{h}<\hat{h}$, a transonic 1-shock wave if $\hat{h} \leq \bar{h}<\bar{h}_{c}$, or a supersonic 1-rarefaction wave if $0<\bar{h}<h_{c} .\left(\bar{U}_{c}=\left(\bar{h}_{c}, \bar{u}_{c}\right) \in S_{1}\left(U_{c}\right)\right.$ with the shock speed $s\left(U_{c}, \bar{U}_{c}\right)=0 ; \quad \hat{U}=(\hat{h}, \hat{u}) \in S_{1}\left(U_{c}\right)$ with $\lambda_{1}(\hat{U})=0$.) The 2-wave, denoted by $\left(\bar{U} ; U_{r}\right)$, is a 2 -shock wave or a 2 -rarefaction wave.

Subcase (c) The wave pattern consists of a supersonic constant state $U^{*}$ in $x<-1$, a stationary wave with 1 -shock $\left(U^{*} ; U_{*}\right)$ at $x_{s 1}=-1$ and another 1 -shock $\left(U_{s 2} ; \bar{U}_{s 2}\right)$ at $x_{s 2}, 0<x_{s 2} \leq 1$ in the region $-1 \leq x \leq 1$ and a 2 -wave in $x>1\left(\bar{U}_{s 2}=\left(\bar{h}_{s 2}, \bar{u}_{s 2}\right) \in S_{1}\left(U_{s 2}\right)\right.$ with the shock speed $\left.s\left(U_{s 2}, \bar{U}_{s 2}\right)=0\right)$. The stationary wave is transonic in $-1 \leq x \leq 0$, attaining the sonic state $U_{0}$ at $x=0$, supersonic at $0<x<x_{s 2}$ followed by a transonic 1-shock $\left(U_{s 2} ; \bar{U}_{s 2}\right)$ at $x=x_{s 2}$, and subsonic in $x_{s 2}<x \leq 1$, attaining the state $U_{1}=\left(h_{1}, u_{1}\right)$ with $\bar{h}_{c} \leq h_{1} \leq h_{*}$ at $x=1$. The 2 -wave $\left(U_{1} ; U_{r}\right)$ is a 2 -shock wave or a 2-rarefaction wave.

Let $T=\left(h_{T}, 0\right)$ be the intersection of $S_{1}\left(U_{l}\right)$ and the $h$-axis and let $Q=\left(h_{Q}, u_{Q}\right)$ be the state that the stationary wave, with 1-shock at the top of the bump $x=0$, attains at $x=1$. The following lemma characterizes the relative positions of $T$ and $Q$ for $h_{l}$ close to $h_{0}$. When the height $r$ of the bump is small, $h_{*}, h_{c}$, and $h^{*}$ are close to $h_{0}$. It turns out that the lemma can be applied to all of region II and part of region I for small $r$. 
Lemma 3.3. There exists a constant $\bar{\theta}$ with $\frac{3}{2}<\bar{\theta}$ such that $h_{Q}<h_{T}$ for all upstream states $U_{l}=\left(h_{l}, u_{l}\right)$ with $0<h_{l} \leq h_{c}$ and $\left(\frac{h_{0}}{h_{l}}\right)^{3}<\bar{\theta}$.

Proof. Since $T \in S_{1}\left(U_{l}\right)=\left\{(h, u): u=u_{l}-\sqrt{\frac{g\left(h+h_{l}\right)}{2 h h_{l}}}\left(h-h_{l}\right), h>h_{l}\right\}$,

$$
\begin{gathered}
0=\frac{C_{0}}{h_{l}}-\sqrt{\frac{g\left(h_{T}+h_{l}\right)}{2 h_{T} h_{l}}}\left(h_{T}-h_{l}\right) \\
\Rightarrow \quad\left(\frac{h_{T}}{h_{l}}\right)^{3}-\left(\frac{h_{T}}{h_{l}}\right)^{2}-\left(2\left(\frac{h_{0}}{h_{l}}\right)^{3}+1\right)\left(\frac{h_{T}}{h_{l}}\right)+1=0 .
\end{gathered}
$$

Since $Q$ is defined as the state that the stationary wave, with 1-shock at the top of the bump $x=0$, attains at $x=1$, we have from (2.3) and (2.4)

$$
\left(\frac{C_{0}^{2}}{2} \frac{1}{\bar{h}^{2}}+g \bar{h}\right)-\left(\frac{C_{0}^{2}}{2} \frac{1}{h_{l}^{2}}+g h_{l}\right)=-g(r-0)
$$

and

$$
\left(\frac{C_{0}^{2}}{2} \frac{1}{h_{Q}^{2}}+g h_{Q}\right)-\left(\frac{C_{0}^{2}}{2} \frac{1}{\tilde{h}^{2}}+g \tilde{h}\right)=-g(0-r)
$$

where $\bar{h}:=h\left(x=0^{-}\right)$and $\tilde{h}:=h\left(x=0^{+}\right)$. Adding (3.2) and (3.3) together, we get

$$
\left(\frac{C_{0}^{2}}{2} \frac{1}{h_{Q}^{2}}+g h_{Q}\right)-\left(\frac{C_{0}^{2}}{2} \frac{1}{h_{l}^{2}}+g h_{l}\right)=-\frac{C_{0}^{2}}{2}\left(\frac{1}{\bar{h}^{2}}-\frac{1}{\tilde{h}^{2}}\right)-g(\bar{h}-\tilde{h}) .
$$

Moreover, the Rankine-Hugoniot condition requires $f(\bar{h})=f(\tilde{h})$, which is equivalent to

$$
C_{0}^{2}\left(\frac{1}{\bar{h}}-\frac{1}{\tilde{h}}\right)=\frac{1}{2} g\left(\tilde{h}^{2}-\bar{h}^{2}\right) .
$$

Combining (3.4) and (3.5), we have

$$
\begin{aligned}
\left(\frac{C_{0}^{2}}{2} \frac{1}{h_{Q}^{2}}+g h_{Q}\right)-\left(\frac{C_{0}^{2}}{2} \frac{1}{h_{l}^{2}}+g h_{l}\right) & =g(\tilde{h}-\bar{h})\left[\frac{-1}{4}\left(2+\frac{\tilde{h}}{\bar{h}}+\frac{\bar{h}}{\tilde{h}}\right)+1\right] \\
& \leq 0
\end{aligned}
$$

which implies

$$
2\left(\frac{h_{l}}{h_{0}}\right)^{3}\left(\frac{h_{Q}}{h_{l}}\right)^{2}-\left(\frac{h_{Q}}{h_{l}}\right)-1 \leq 0 .
$$

So

$$
\begin{aligned}
\frac{h_{Q}}{h_{l}} & \leq \frac{1+\sqrt{1+8\left(\frac{h_{l}}{h_{0}}\right)^{3}}}{4\left(\frac{h_{l}}{h_{0}}\right)^{3}} \\
& =\frac{1}{4}\left[\left(\frac{h_{0}}{h_{l}}\right)^{3}+\sqrt{\left(\frac{h_{0}}{h_{l}}\right)^{6}+8\left(\frac{h_{0}}{h_{l}}\right)^{3}}\right] \\
& =\frac{1}{4}\left[\theta+\sqrt{\theta^{2}+8 \theta}\right]
\end{aligned}
$$

with $\theta:=\left(\frac{h_{0}}{h_{l}}\right)^{3}, 1 \leq \theta<\infty$. On the other hand, it is easy to check that

$$
\beta(y) \equiv y^{3}-y^{2}-(1+2 \theta) y+1=0
$$


has one negative zero and two positive zeros, say $z_{1}$ and $z_{2}$, with $z_{1}<1<z_{2}$. (3.1) tells us that $\frac{h_{T}}{h_{l}}(>1)$ is one zero of $\beta(y)=0$, and $(1+3 \sqrt{1+3(1+2 \theta)}) / 3$ $\left(<\frac{h_{T}}{h_{l}}\right)$ is the local minimum point of $\beta(y)$. Thus, if $\theta$ satisfies

$$
\frac{1+3 \sqrt{1+3(1+2 \theta)}}{3}>\frac{1}{4}\left(\theta+\sqrt{\theta^{2}+8 \theta}\right)
$$

then $h_{T}>h_{Q}$.

To estimate such $\theta$, we have from (3.6)

$$
5+6 \theta+2 \sqrt{4+6 \theta}>\frac{9}{16}\left(\theta+\sqrt{\theta^{2}+8 \theta}\right)^{2} .
$$

Let $\Gamma(\theta)=5+6 \theta+2 \sqrt{4+6 \theta}-\frac{9}{16}\left(\theta+\sqrt{\theta^{2}+8 \theta}\right)^{2}$. Then

$$
\begin{aligned}
\Gamma^{\prime}(\theta) & <6+\frac{6}{\sqrt{4+6 \theta}}-\frac{9}{8}\left(\theta+\sqrt{\theta^{2}+8 \theta}\right)-\frac{9}{8}(\theta+4) \\
\Rightarrow \quad \Gamma^{\prime}(\theta) & <0 \quad \forall \quad \theta \geq 1,
\end{aligned}
$$

that is, $\Gamma(\theta)$ is a decreasing function for $\theta \geq 1$. Since $\Gamma(1), \Gamma(3 / 2)>0$, and $\Gamma(2)<0$, we know that there exists $\bar{\theta}>3 / 2$ such that (3.4) holds for $1 \leq \theta<\bar{\theta}$.

TheOREm 3.4. Let $0<h^{*}<h_{c}<h_{0}<h_{*}$ be the quantities defined in Sec. 2 and $U_{l}=\left(h_{l}, u_{l}\right), 0<h_{l}<h^{*}$, be the given upstream state for the system of shallow water equations (1.1)(1.2). Assume the height $r$ of the bump is small enough and $h_{l}$ is close to $h_{0}$. Then the possible asymptotic wave patterns are as follows: for the downstream state $U_{r}$ lying in (see Fig. 8):

1. the open set $\Sigma_{1}$ with boundaries $R_{2}\left(M_{1}\right), \overline{O M_{1}}, \overline{O L_{1}}$, and $R_{2}\left(\bar{U}_{l}\right) \cup\left\{\bar{U}_{l}\right\} \cup S_{2}\left(\bar{U}_{l}\right)$, there is 1 wave pattern described in Subcase (i) of Case 3.1;

2. the open set $\Sigma_{2}$ with boundaries $R_{2}(Q) \cup\{Q\} \cup S_{2}(Q), \overline{L_{2} T}$, and $R_{2}(T)$, there is 1 wave pattern described in Subcase (ii) of Case 3.1;

3. $R_{2}\left(\bar{U}_{l}\right) \cup\left\{\bar{U}_{l}\right\} \cup S_{2}\left(\bar{U}_{l}\right)$, there are 2 wave patterns described in Subcase (iii) of Case 3.1 with transonic stationary 1 -shocks at $x_{s}=-1$ and $x_{s}=1$, respectively;

4. $R_{2}(Q) \cup\{Q\} \cup S_{2}(Q)$, there are 2 wave patterns; one is described in Subcase (ii) of Case 3.1 and the other is described in Subcase (iii) of Case 3.1 with transonic stationary 1-shock at $x_{s}=0$;

5. the open set $\Sigma_{3}$ with boundaries $R_{2}\left(\bar{U}_{l}\right) \cup \bar{U}_{l} \cup S_{2}\left(\bar{U}_{l}\right), \overline{L_{1} L_{2}}$, and $R_{2}(Q) \cup\{Q\} \cup$ $S_{2}(Q)$, there are 3 wave patterns; one is described in Subcase (ii) of Case 3.1 and the others are described in Subcase (iii) of Case 3.1 with transonic stationary 1 -shocks at $x_{s}$ and $-x_{s}, 0<\left|x_{s}\right|<1$, respectively.

Moreover, when $h_{l}=h^{*}$, the possible wave patterns are as follows: for the downstream state $U_{r}$ lying in (see Fig. 9):

1. $R_{2}\left(M_{0}\right)$ or the open set $\Sigma_{12}$ with boundaries $R_{2}\left(M_{1}\right), \overline{M_{0} M_{1}}$, and $R_{2}\left(M_{0}\right)$, there is 1 wave pattern described as Subcase (i) of Case 3.1;

2. the open set $\Sigma_{2}$ with boundaries $R_{2}(T), R_{2}(Q) \cup\{Q\} \cup S_{2}(Q)$, and $\overline{L_{2} T}$, there is 1 wave pattern described as Subcase (ii) of Case 3.1; 


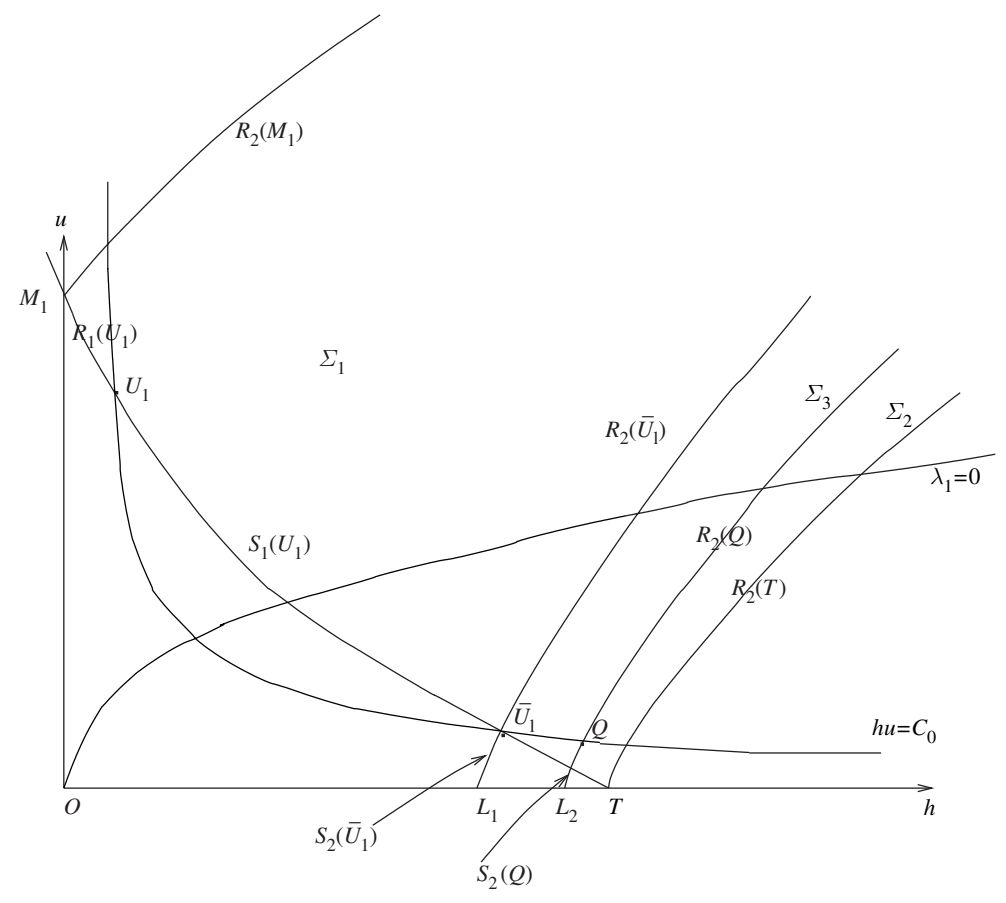

FIG. 8.

3. the open set $\Sigma_{10}$ with boundaries $R_{2}\left(M_{0}\right), \overline{O M_{0}}, \overline{O L_{0}}$, and $R_{2}\left(\bar{U}_{c}\right) \cup\left\{\bar{U}_{c}\right\} \cup$ $S_{2}\left(\bar{U}_{c}\right)$, there are 2 wave patterns described as Subcase (i) of Case 3.1 and choke + Subcase $(b)$ in Case 3.2 ;

4. $R_{2}\left(\bar{U}_{c}\right) \cup\left\{\bar{U}_{c}\right\} \cup S_{2}\left(\bar{U}_{c}\right)$ and the open set $\Sigma_{11}$ with boundaries $R_{2}\left(\bar{U}_{c}\right) \cup\left\{\bar{U}_{c}\right\} \cup$ $S_{2}\left(\bar{U}_{c}\right), \overline{L_{0} L_{1}}$, and $R_{2}\left(U_{*}\right) \cup\left\{U_{*}\right\} \cup S_{2}\left(U_{*}\right)$, there are 2 wave patterns described as Subcase (i) of Case 3.1 and choke + Subcase (c) in Case 3.2;

5. $R_{2}\left(U_{*}\right) \cup\left\{U_{*}\right\} \cup S_{2}\left(U_{*}\right)$, there are 2 wave patterns described as Subcase (iii) of Case 3.1 and choke + Subcase $(a)$ in Case 3.2;

6. $R_{2}(Q) \cup\{Q\} \cup S_{2}(Q)$, there are 2 wave patterns described as Subcase (ii) and Subcase (iii) of Case 3.1;

7. the open set $\Sigma_{3}$ with boundaries $R_{2}\left(U_{*}\right) \cup\left\{U_{*}\right\} \cup S_{2}\left(U_{*}\right), \overline{L_{1} L_{2}}$, and $R_{2}(Q) \cup$ $\{Q\} \cup S_{2}(Q)$, there are 3 wave patterns described as Subcase (ii) and Subcase (iii) of Case 3.1.

Case 3.3: For $U_{l} \in I I$ and $h^{*}<h_{l}<h_{c}$, we have the following possible asymptotic wave patterns:

Subcase (i) The wave pattern consists of a supersonic constant state $U_{l}=\left(h_{l}, u_{l}\right)$ in $x \leq-1$, a stationary wave in $-1 \leq x \leq 1$, and a 1 -wave with positive speed followed by a 2 -wave in the region $x \geq 1$. In this case, the possible 


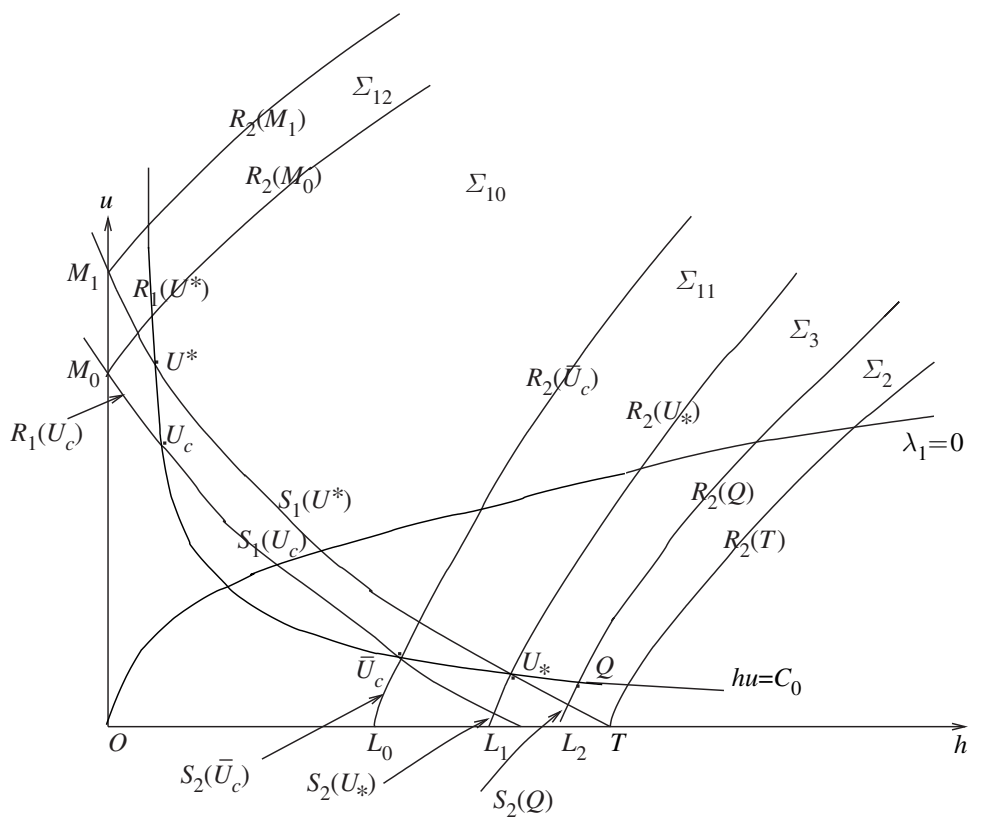

FIG. 9.

asymptotic wave patterns are the same as Subcase (i) in Case 3.1.

Subcase (ii) The wave pattern consists of a 1-shock in $x<-1$, a stationary wave in $-1 \leq x \leq 1$, and either a 2 -wave or a 1 -wave followed by a 2 -wave in $x>1$. The 1-shock, denoted by $\left(U_{l} ; \bar{U}\right)\left(\bar{U}=(\bar{h}, \bar{u}) \in S_{1}\left(U_{l}\right)\right.$ with $\left.\bar{h} \geq \tilde{h}_{*}\right)$ is transonic, where $\left(\tilde{h}_{*}, \tilde{u}_{*}\right)=\tilde{U}_{*} \in S_{1}\left(U_{l}\right)$ such that there exists a stationary wave depending on the parameter $\tilde{C}=\tilde{h}_{*} \tilde{u}_{*}$ and attaining sonic state $\left(\tilde{h}_{0}=\right.$ $\left.g^{-1 / 3} \tilde{C}^{2 / 3}, \tilde{u}_{0}=\tilde{C} / \tilde{h}_{0}\right)$ at the top of the bump $x=0$. The assumption of existence $\tilde{U}_{*}$ holds when the height $r$ of the bump is small enough. The stationary wave patterns are richer for $\bar{h}=\tilde{h}_{*}$ than that for $\bar{h}>\tilde{h}_{*}$ :

(1) for $\bar{h}>\tilde{h}_{*}$, the stationary wave is continuous and subsonic, and attains $\bar{U}$ at $x=1^{-}$. The 2 -wave is a 2 -rarefaction wave or a 2 -shock wave (see Fig. 10-1).

(2) for $\bar{h}=\tilde{h}_{*}$, the stationary wave attains the sonic state $\tilde{U}_{0}=\left(\tilde{h}_{0}, \tilde{u}_{0}\right)$ at $x=0$, and then there are stationary wave patterns similar with Subcases $(\mathrm{a})(\mathrm{b})(\mathrm{c})$ in Case 3.2 (see Fig. 10-2).

Subcase (a) the stationary wave with choke is continuous and subsonic, and attains the state $\tilde{U}_{*}$ at $x=1$. The 2 -wave is a 2 -rarefaction wave or a 2-shock wave. 
Subcase (b) the stationary wave with choke is continuous, subsonic in $-1 \leq$ $x<0$ and supersonic in $0<x \leq 1$, attaining the state $\tilde{U}_{c}$ at $x=1$. The 1 -wave, denoted by $\left(\tilde{U}_{c} ; \overline{\tilde{U}}\right)$, is either a supersonic 1-shock wave if $\tilde{h}_{c}<\overline{\tilde{h}}<\hat{\tilde{h}}_{c}$, a transonic 1-shock wave if $\hat{\tilde{h}}_{c} \leq \overline{\tilde{h}}<\overline{\tilde{h}}_{c}$, or a supersonic 1-rarefaction wave if $0<\overline{\tilde{h}}<\tilde{h}_{c}$. $\left(\overline{\tilde{U}}_{c}=\left(\tilde{\tilde{h}}_{c}, \tilde{\tilde{u}}_{c}\right) \in S_{1}\left(\tilde{U}_{c}\right)\right.$ with the shock speed $s\left(\tilde{U}_{c}, \overline{\tilde{U}}_{c}\right)=0$; $\hat{\tilde{U}}_{c}=\left(\hat{\tilde{h}}_{c}, \hat{\tilde{u}}_{c}\right) \in S_{1}\left(\tilde{U}_{c}\right)$ with $\lambda_{1}\left(\hat{\tilde{h}}_{c}, \hat{\tilde{u}}_{c}\right)=0$.) The 2-wave, denoted by $\left(\overline{\tilde{U}} ; U_{r}\right)$, is a 2 -shock wave or a 2 -rarefaction wave.

Subcase (c) the stationary wave with choke and a transonic 1-shock, denoted by $\left(U_{s 2} ; \bar{U}_{s 2}\right)$, at $x_{s 2}, 0<x_{s 2} \leq 1$, is subsonic in $-1 \leq x<0$ or $x_{s 2}<x \leq 1$, and supersonic in $0<x<x_{s 2}$. The 2 -wave $\left(U_{1} ; U_{r}\right)$ in $x>1$ is 2 -rarefaction wave or a 2 -shock wave.

Subcase (iii) The wave pattern consists of a supersonic constant state $U_{l}$ in $x<-1$, a stationary wave with 1 -shock at $x_{s}, y<x_{s} \leq 1$ (for some $y$ ), in the region $-1 \leq x \leq 1$, and a 2 -wave in $x>1$, or of a supersonic constant state $U_{l}$ in $x<-1$, a stationary wave with 1 -shock at $y$ and choke in $-1 \leq x \leq 1$, and either a 1 -wave followed by a 2 -wave or a 2 -wave in $x>1$. The point $y$ depends on $h_{l}$ such that there exists a stationary wave with initial value $h_{l}$ and one transonic 1 -shock just at $y$ attaining the sonic state $U_{0}$ at $x=0$. Let $h(x)$ and $\tilde{h}(x)$ be the continuous stationary solutions on $[-1,0]$ with initial
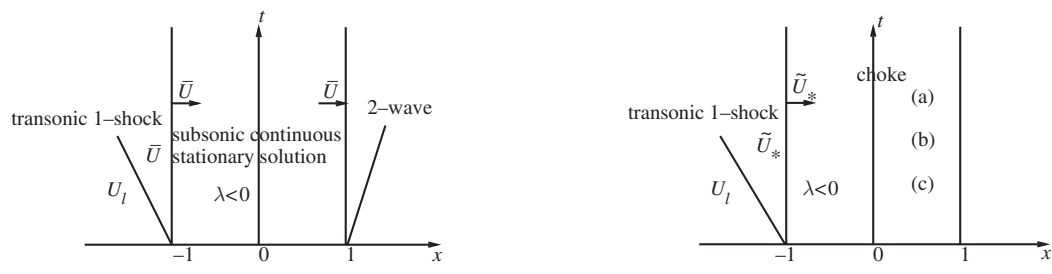

FIG. 10-1 FIG. 10-2

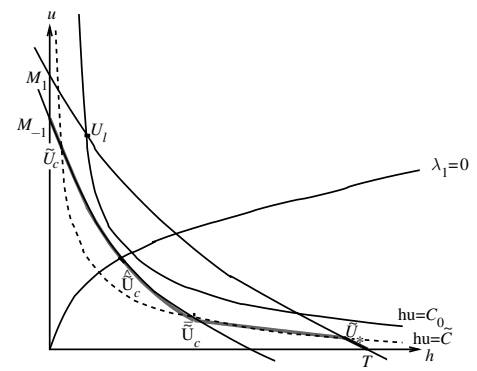

FIG. 10-3 

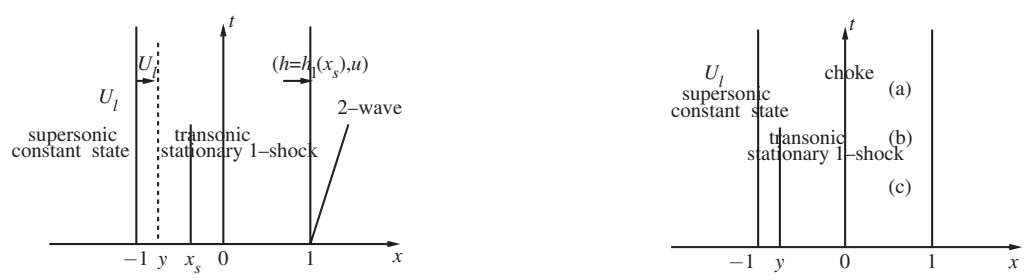

FIG. 11-1

FIG. 11-2

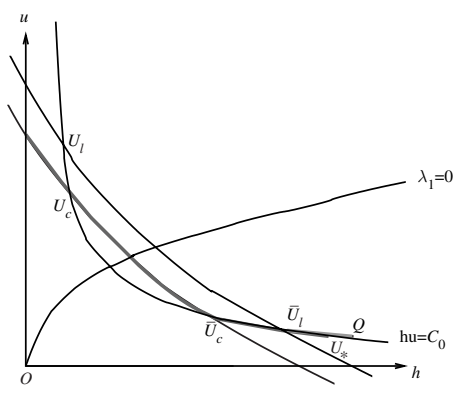

FIG. 11-3

values $h_{l}$ and $h_{*}$ at $x=-1$, respectively. Therefore, $y$ can be reinterpreted in the following lemma.

Lemma 3.5. Suppose that $h^{*}<h_{l}<h_{c}$ and $h(x), \tilde{h}(x)$ are continuous solutions of the stationary equation $(2.3)(2.4)$ with the initial values $h_{l}$ and $h_{*}$ at $x=-1$, respectively. Then there is a unique point $y \in(-1,0)$, depending on $h_{l}$, such that $\overline{h(y)}=\tilde{h}(y)$ where $\overline{h(y)}$ satisfying $f(h(y))=f(\overline{h(y)})$.

Moreover, $y\left(h_{l}\right)$ is an increasing function of $h_{l}$ from $\left(h^{*}, h_{c}\right)$ onto $(-1,0)$ (if $B_{x}$ is decreasing on $(-1,0))$.

Proof. Since $\tilde{h}(x), \overline{h(x)}=\frac{h(x)}{2}\left(\sqrt{1+\frac{8 C_{0}^{2}}{g h(x)^{3}}}-1\right)$ are continuous on $[-1,0]$ and $\overline{h(-1)}=$ $\bar{h}_{l}<h_{*}=\tilde{h}(-1), \overline{h(0)}>h_{0}=\tilde{h}(0)$, there exists a $y \in(-1,0)$ such that $\tilde{h}(y)=\overline{h(y)}$. The uniqueness of $y$ is a consequence of the Key Lemma.

Suppose $h^{*}<h_{1}<h_{2}<h_{c}$ and $h_{1}(x), h_{2}(x)$ are both continuous stationary solutions of the stationary equation $(2.3)(2.4)$ with initial values $h_{1}(-1)=h_{1}, h_{2}(-1)=h_{2}$ respectively. Let $y_{1}=y\left(h_{1}\right), y_{2}=y\left(h_{2}\right)$. It remains to show that $y_{1}<y_{2}$. If $y_{1}=y_{2}$, then $h_{1}\left(y_{1}\right)<h_{2}\left(y_{2}\right)$ and so $\overline{h_{1}\left(y_{1}\right)}>\overline{h_{2}\left(y_{2}\right)}$. But by definition of $y_{1}$ and $y_{2}, \overline{h_{1}\left(y_{1}\right)}=\tilde{h}\left(y_{1}\right)=$ $\tilde{h}\left(y_{2}\right)=\overline{h_{2}\left(y_{2}\right)}$, a contradiction. If $y_{1}>y_{2}$, then $\overline{h_{1}\left(y_{1}\right)}=\tilde{h}\left(y_{1}\right)<\tilde{h}\left(y_{2}\right)=\overline{h_{2}\left(y_{2}\right)}$ and so $h_{1}\left(y_{1}\right)>h_{2}\left(y_{2}\right)$. Let $x_{1} \in(-1,0)$ such that $h_{1}(x)$ has value $h_{2}\left(y_{2}\right)$ at $x_{1}$. Then $x_{1}>y_{2}$. On the other hand, by the Key Lemma, we have $y_{1}-y_{2}<y_{1}-x_{1}$ and hence $x_{1}<y_{2}$, a contradiction (see Fig. 12). 


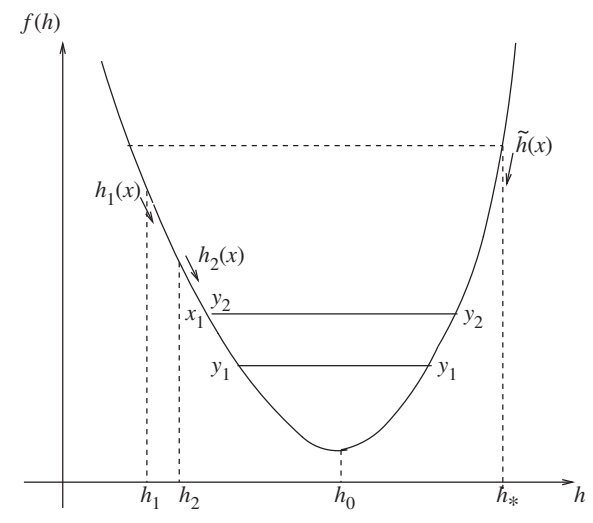

FIG. 12 .

With regard to the position of the stationary 1-shock, there are different possible wave patterns as follows:

(1) the wave pattern consists of a supersonic constant state $U_{l}$ in $x<-1$, a stationary wave with 1 -shock at $x_{s}, y<x_{s} \leq 1$, and a 2 -wave in $x>1$. The stationary wave with 1 -shock is transonic and the 2 -wave is a 2 -rarefaction wave or a 2 -shock wave. The asymptotic wave patterns are the same as those in Subcase (iii) of Case 3.1 (see Fig. 11-1).

(2) The wave pattern consists of a supersonic constant state $U_{l}$ in $x<-1$, a stationary wave with 1-shock at $y$ and choke in $-1 \leq x \leq 1$, and either a 1-wave followed by a 2 -wave or a 2 -wave in $x>1$. The wave patterns are the same as those Subcases (a)(b)(c) for $U_{l}=U^{*}$ in Case 3.2 except the position of the transonic stationary 1-shock (see Fig. 11-2).

Case 3.4: $U_{l} \in I I$ and $h_{l}=h_{c}$.

Comparing with the wave patterns in Case 3.3, the possible wave patterns of Subcases (i) and (iii) become the wave pattern of Subcase (i) in this case:

Subcase (i) The wave pattern consists of a supersonic constant state $U_{c}$ in $x<-1$, a stationary wave with choke in $-1 \leq x \leq 1$, and either a 2 -wave or a 1 -wave followed by a 2 -wave in $x>1$. More precisely,

Subcase (a) the wave pattern consists of a supersonic constant state $U_{c}$ in $x<-1$, a stationary wave with choke in $-1 \leq x \leq 1$, and a 2 -wave in $x>1$. The stationary wave with choke is continuous, supersonic in $-1 \leq x<0$, and subsonic in $0<x \leq 1$, and attains the state $U_{*}$ at $x=1$. The 2 -wave is a 2 -rarefaction wave or a 2 -shock wave. 
Subcase (b) the wave pattern consists of a supersonic constant state $U_{c}$ in $x<-1$, a stationary wave with choke in $-1 \leq x \leq 1$, and a 1-wave followed by a 2 -wave in $x>1$. The stationary wave with choke is continuous, supersonic in $-1 \leq x<0$ or $0<x \leq 1$, and attains the state $U_{c}$ at $x=1^{-}$. The 1 -wave is either a supersonic 1 -shock, a transonic 1-shock, or a supersonic 1-rarefaction. The 2-wave is a 2-rarefaction wave or a 2 -shock wave.

Subcase (c) the wave pattern consists of a supersonic constant state $U_{c}$ in $x<-1$, a stationary wave with choke and a 1 -shock in $-1 \leq x \leq 1$, and a 2 wave in $x>1$. The 1 -shock at $x_{s}, 0<x_{s}<1$, is transonic.

Subcase (ii) the wave patterns are the same as those in Subcase (ii) of Case 3.3:

(1) the wave pattern consists of a 1 -shock $\left(U_{c} ; \bar{U}\right), \bar{h}>\tilde{h}_{*}$ in $x<-1$, a stationary wave in $-1 \leq x \leq 1$, and a 2 -wave in $x>1$. The stationary wave is continuous and subsonic, and attains $\bar{U}$ at $x=1^{-}$. The 2 -wave is a 2 -rarefaction wave or a 2 -shock wave.

(2) the wave pattern consists of a 1 -shock $\left(U_{c} ; \tilde{U}_{*}\right)$ in $x<-1$, a stationary wave with choke in $-1 \leq x \leq 1$, and either a 2 -wave or a 1 -wave followed by a 2 -wave in $x>1$.

If the height $r$ of the bump is small enough, then $U_{*}(C), U_{c}(C), U^{*}(C)$, and $\bar{U}_{c}(C)$ for all $C_{0} \leq C \leq \tilde{C}$ are close to $U_{0}=\left(h_{0}, u_{0}\right)$, and the curves $\left\{\left(h_{*}(C), u_{*}(C)\right)\right\}$, $\left\{\left(h_{c}(C), u_{c}(C)\right)\right\}$, and $\left\{\left(h^{*}(C), u^{*}(C)\right)\right\}$ have the tangent slopes approximating to the tangent slope of the curve $\left\{\left(h_{0}(C), u_{0}(C)\right)\right\}$; the tangent slope is less than the slope of the eigenvector $r_{2}\left(h_{0}(C), u_{0}(C)\right)$ corresponding to $\lambda_{2}$. Then all 2-rarefaction curves $R_{2}$ of states on those curves mentioned above do not intersect with each other. Also, the Hugoniot curves, $S_{2}\left(U_{*}\right)$ and $S_{2}\left(\tilde{U}_{*}\right), S_{2}\left(\bar{U}_{c}\right)$ and $S_{2}\left(\overline{\tilde{U}}_{c}\right)$, do not intersect mutually. Therefore, the wave patterns can be precisely described for given upstream state $U_{l}$ and downstream state $U_{r}$.

TheOREm 3.6. Let $U_{l}=\left(h_{l}, u_{l}\right), h^{*}<h_{l}<h_{c}$, be the given upstream state for the system of shallow water equations (1.1)(1.2). Under the assumption that the height $r$ of the bump is small enough, the time-asymptotic wave patterns are as follows: for the downstream state $U_{r}$ lying in (see Fig. 13):

1. $R_{2}\left(M_{0}\right)$ or the open set $\Sigma_{11}$ with boundaries $R_{2}\left(M_{1}\right), \overline{M_{1} M_{0}}$, and $R_{2}\left(M_{0}\right)$, there is 1 wave pattern described in Subcase (i);

2. $R_{2}\left(M_{-1}\right)$ or the open set $\Sigma_{12}$ with boundaries $R_{2}\left(M_{0}\right), \overline{M_{0} M_{-1}}$, and $R_{2}\left(M_{-1}\right)$, there are 2 wave patterns described in Subcase (i) and choke $+(2)(b)$ of Subcase (iii) respectively; 


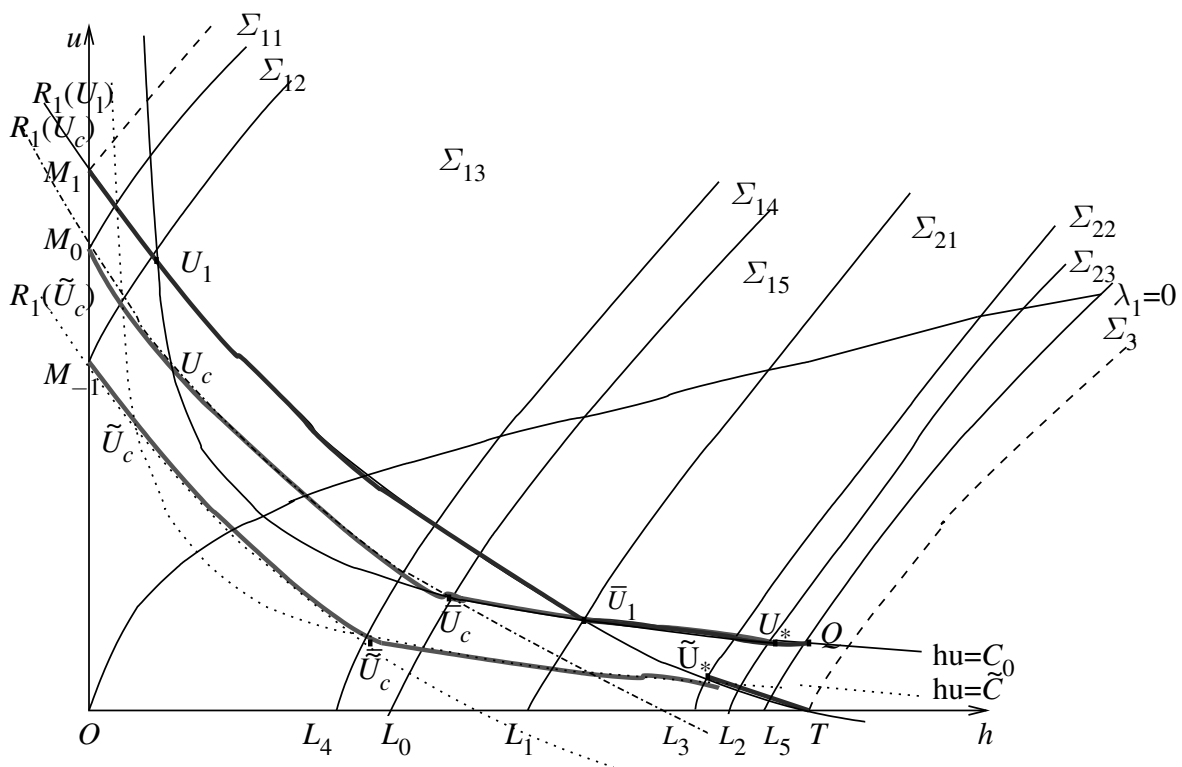

FIG. 13.

3. the open set $\Sigma_{13}$ with boundaries $R_{2}\left(M_{-1}\right), \overline{O M_{-1}}, \overline{O L_{4}}$, and $R_{2}\left(\overline{\tilde{U}}_{c}\right) \cup\left\{\overline{\tilde{U}}_{c}\right\} \cup$ $S_{2}\left(\overline{\tilde{U}}_{c}\right)$, there are 3 wave patterns described in Subcase (i), choke $+(2)(b)$ of Subcase (ii), and choke $+(2)(b)$ of Subcase (iii) respectively;

4. $\quad R_{2}\left(\overline{\tilde{U}}_{c}\right) \cup\left\{\overline{\tilde{U}}_{c}\right\} \cup S_{2}\left(\overline{\tilde{U}}_{c}\right)$ or the open set $\Sigma_{14}$ with boundaries $R_{2}\left(\overline{\tilde{U}}_{c}\right) \cup\left\{\overline{\tilde{U}}_{c}\right\} \cup$ $S_{2}\left(\bar{U}_{c}\right), \overline{L_{4} L_{0}}$, and $R_{2}\left(\bar{U}_{c}\right) \cup\left\{\bar{U}_{c}\right\} \cup S_{2}\left(\bar{U}_{c}\right)$, there are 3 wave patterns described in Subcase (i), choke $+(2)(c)$ of Subcase (ii), and choke $+(2)(b)$ of Subcase (iii) respectively;

5. $R_{2}\left(\bar{U}_{c}\right) \cup\left\{\bar{U}_{c}\right\} \cup S_{2}\left(\bar{U}_{c}\right)$ and the open set $\Sigma_{15}$ with boundaries $R_{2}\left(\bar{U}_{c}\right) \cup\left\{\bar{U}_{c}\right\} \cup$ $S_{2}\left(\bar{U}_{c}\right), \overline{L_{0} L_{1}}$, and $R_{2}\left(\bar{U}_{l}\right) \cup\left\{\bar{U}_{l}\right\} \cup S_{2}\left(\bar{U}_{l}\right)$, there are 3 wave patterns described in Subcase (i), choke $+(2)(c)$ of Subcase (ii), and choke $+(2)(c)$ of Subcase (iii) respectively;

6. $R_{2}\left(\bar{U}_{l}\right) \cup\left\{\bar{U}_{l}\right\} \cup S_{2}\left(\bar{U}_{l}\right)$ and the open set $\Sigma_{21}$ with boundaries $R_{2}\left(\bar{U}_{l}\right) \cup\left\{\bar{U}_{l}\right\} \cup$ $S_{2}\left(\bar{U}_{l}\right), \overline{L_{1} L_{3}}$, and $R_{2}\left(\tilde{U}_{*}\right) \cup\left\{\tilde{U}_{*}\right\} \cup S_{2}\left(\tilde{U}_{*}\right)$, there are 3 wave patterns described in choke $+(2)(c)$ of Subcase (ii), choke $+(2)(c)$ of Subcase(iii), and (1) of Subcase (iii), respectively;

7. $R_{2}\left(\tilde{U}_{*}\right) \cup\left\{\tilde{U}_{*}\right\} \cup S_{2}\left(\tilde{U}_{*}\right)$, there are 3 wave patterns described in choke $+(2)(a)$ of Subcase (ii), choke $+(2)(c)$ of Subcase (iii), and (1) of Subcase (iii), respectively;

8. the open set $\Sigma_{22}$ with boundaries $R_{2}\left(\tilde{U}_{*}\right) \cup\left\{\tilde{U}_{*}\right\} \cup S_{2}\left(\tilde{U}_{*}\right), \overline{L_{3} L_{2}}$, and $R_{2}\left(U_{*}\right) \cup$ $\left\{U_{*}\right\} \cup S_{2}\left(U_{*}\right)$, there are 3 wave patterns described in (1) of Subcase (ii), choke $+(2)(c)$ of Subcase (iii), and (1) of Subcase (iii) respectively;

9. $R_{2}\left(U_{*}\right) \cup\left\{U_{*}\right\} \cup S_{2}\left(U_{*}\right)$, there are 3 wave patterns described in (1) of Subcase (ii), choke $+(2)(a)$ of Subcase (iii), and (1) of Subcase (iii) respectively; 
10. the open set $\Sigma_{23}$ with boundaries $R_{2}\left(U_{*}\right) \cup\left\{U_{*}\right\} \cup S_{2}\left(U_{*}\right), \overline{L_{2} L_{5}}$, and $R_{2}(Q) \cup$ $\{Q\} \cup S_{2}(Q)$, there are 3 wave patterns described in (1) of Subcase (ii) and (1) in Subcase (iii)with transonic stationary 1-shocks at $x_{s}$ and $-x_{s}, y<x_{s}<0$;

11. $R_{2}(Q) \cup\{Q\} \cup S_{2}(Q)$, there are 2 wave patterns described in (1) of Subcase (ii) and (1) of Subcase (iii);

12. the open set $\Sigma_{3}$ with boundaries $R_{2}(Q) \cup\{Q\} \cup S_{2}(Q), \overline{L_{5} T}$, and $R_{2}(T)$, there is 1 wave pattern described in (1) of Subcase (ii).

When $h_{l}=h_{c}$, the time-asymptotic wave patterns are as follows: for the downstream state $U_{r}$ lying in (see Fig. 14):

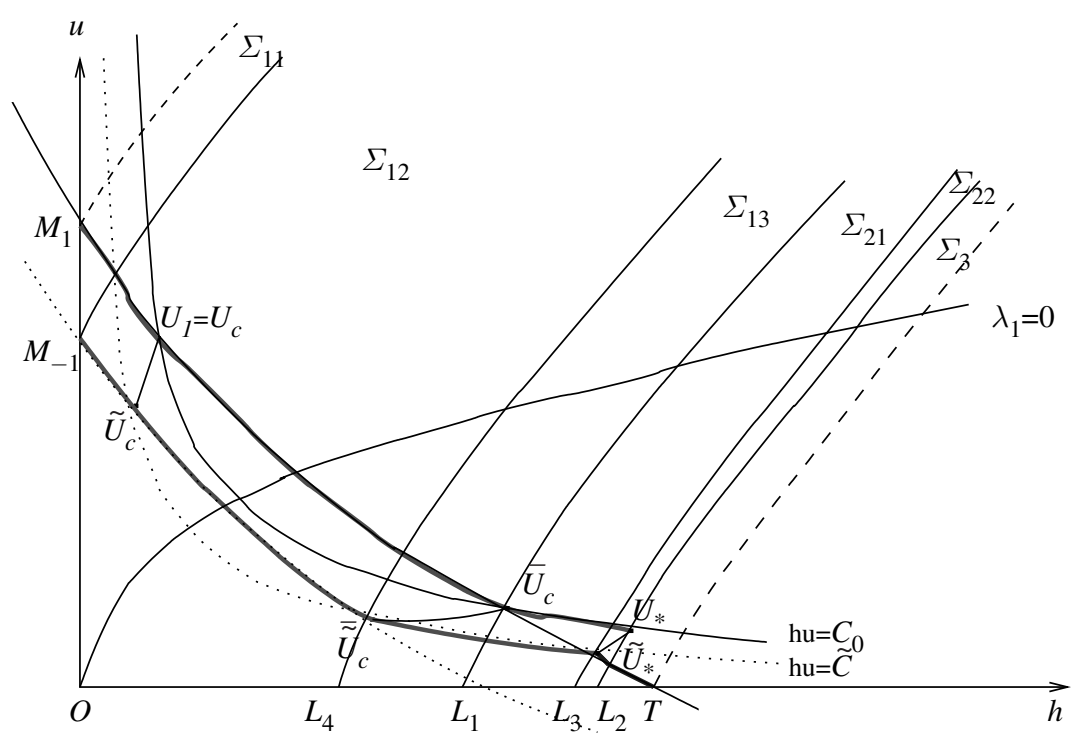

FIG. 14.

1. $R_{2}\left(M_{-1}\right)$ or the open set $\Sigma_{11}$ with boundaries $R_{2}\left(M_{1}\right), \overline{M_{1} M_{-1}}$, and $R_{2}\left(M_{-1}\right)$, there is 1 wave pattern described in choke $+(b)$ of Subcase (i);

2. the open set $\Sigma_{12}$ with boundaries $R_{2}\left(M_{-1}\right), \overline{O M_{-1}}, \overline{O L_{4}}$, and $R_{2}\left(\overline{\tilde{U}}_{c}\right) \cup\left\{\overline{\tilde{U}}_{c}\right\} \cup$ $S_{2}\left(\overline{\tilde{U}}_{c}\right)$, there are 2 wave patterns described in choke $+(b)$ of Subcase (i) and choke $+(2)(b)$ of Subcase (ii);

3. $R_{2}\left(\overline{\tilde{U}}_{c}\right) \cup\left\{\overline{\tilde{U}}_{c}\right\} \cup S_{2}\left(\overline{\tilde{U}}_{c}\right)$ or the open set $\Sigma_{13}$ with boundaries $R_{2}\left(\overline{\tilde{U}}_{c}\right) \cup\left\{\overline{\tilde{U}}_{c}\right\} \cup$ $S_{2}\left(\overline{\tilde{U}}_{c}\right), \overline{L_{4} L_{1}}$, and $R_{2}\left(\bar{U}_{c}\right) \cup\left\{\bar{U}_{c}\right\} \cup S_{2}\left(\bar{U}_{c}\right)$, there are 2 wave patterns described in choke $+(b)$ of Subcase (i) and choke $+(2)(c)$ of Subcase (ii);

4. $R_{2}\left(\bar{U}_{c}\right) \cup\left\{\bar{U}_{c}\right\} \cup S_{2}\left(\bar{U}_{c}\right)$ or the open set $\Sigma_{21}$ with boundaries $R_{2}\left(\bar{U}_{c}\right) \cup\left\{\bar{U}_{c}\right\} \cup$ $S_{2}\left(\bar{U}_{c}\right), \overline{L_{1} L_{3}}$, and $R_{2}\left(\tilde{U}_{*}\right) \cup\left\{\tilde{U}_{*}\right\} \cup S_{2}\left(\tilde{U}_{*}\right)$, there are 2 wave patterns described in choke $+(c)$ of Subcase (i) and choke $+(2)(c)$ of Subcase (ii);

5. $R_{2}\left(\tilde{U}_{*}\right) \cup\left\{\tilde{U}_{*}\right\} \cup S_{2}\left(\tilde{U}_{*}\right)$, there are 2 wave patterns described in choke $+(c)$ of Subcase (i) and choke $+(2)(a)$ of Subcase (ii); 
6. the open set $\Sigma_{22}$ with boundaries $R_{2}\left(\tilde{U}_{*}\right) \cup\left\{\tilde{U}_{*}\right\} \cup S_{2}\left(\tilde{U}_{*}\right), \overline{L_{3} L_{2}}$ and $R_{2}\left(U_{*}\right) \cup$ $\left\{U_{*}\right\} \cup S_{2}\left(U_{*}\right)$, there are 2 wave patterns described in choke $+(c)$ of Subcase (i) and (1) of Subcase (ii);

7. $R_{2}\left(U_{*}\right) \cup\left\{U_{*}\right\} \cup S_{2}\left(U_{*}\right)$, there are 2 wave patterns described in choke $+(a)$ of Subcase (i) and (1) of Subcase (ii);

8. the open set $\Sigma_{3}$ with boundaries $R_{2}\left(U_{*}\right) \cup\left\{U_{*}\right\} \cup S_{2}\left(U_{*}\right), \overline{L_{2} T}$, and $R_{2}(T)$, there is 1 wave pattern described in (1) of Subcase (ii).

Case 3.5: For $U_{l} \in I I I$.

For $h_{c}<h_{l}<h_{0}$ and the assumption of the small height $r$ of the bump, we have the following possible wave patterns:

Subcase (1) the wave pattern consists of a transonic 1-shock $\left(U_{l} ; \bar{U}\right),\left(\bar{h}>\tilde{h}_{*}\right)$ in $x<$ -1 , a stationary wave in $-1 \leq x \leq 1$, and a 2 -wave in $x>1$. The stationary wave is continuous and subsonic, and attains $\bar{U}$ at $x=1^{-}$. The 2 -wave is a 2-rarefaction wave or a 2-shock wave; or

Subcase (2) the wave pattern consists of a transonic 1 -shock $\left(U_{l} ; \tilde{U}_{*}\right)$ in $x<-1$, a stationary wave with choke in $-1 \leq x \leq 1$, and either a 2 -wave or a 1 -wave followed by a 2 -wave. The stationary wave is one of those (a)(b)(c) of Subcase (ii)(2) in Case 3.3.

Case 3.6: For $U_{l} \in I V, h_{0}<h_{l}<h_{*}$, and the assumption of the small height of the bump:

Subcase (1) The wave pattern consists of a subsonic 1-shock $\left(U_{l} ; \bar{U}\right),\left(\bar{h}>\tilde{h}_{*}\right)$ in $x<$ -1 , a stationary wave in $-1 \leq x \leq 1$, and a 2 -wave in $x>1$; or

Subcase (2) the wave pattern consists of a subsonic 1 -shock $\left(U_{l} ; \tilde{U}_{*}\right)$ in $x<-1$, a stationary wave with choke in $-1 \leq x \leq 1$, and either a 2 -wave or a 1 -wave followed by a 2 -wave. The stationary wave is one of those (a)(b)(c) of Subcase (ii)(2) in Case 3.3 .

Case 3.7 For $U_{l} \in I V$ with $h_{l}=h_{*}$, there exist possible asymptotic wave patterns as follows:

Subcase (i) The wave pattern consists of a subsonic constant state $U_{*}$ in $x<-1$, a stationary wave with choke in $-1 \leq x \leq 1$, and either a 2 -wave or a 1 -wave followed by a 2 -wave in $x>1$. The stationary wave is one of those (a)(b)(c) of Subcase (ii)(2) in Case 3.3.

Subcase (ii) The wave pattern consists of a subsonic 1 -shock $\left(U_{*} ; \bar{U}\right), \bar{h}>h_{*}$ in $x<-1$, a stationary wave in $-1 \leq x \leq 1$, and a 2 -wave in $x>1$. The stationary 
wave is continuous and subsonic, and attains the state $\bar{U}$ at $x=1^{-}$. The 2 -wave is a 2 -rarefaction wave or a 2 -shock wave.

REMARK 3.1. For given upstream state $U_{l}$ in Cases 3.4, 3.5, 3.6, and 3.7, and downstream state $U_{r}$ under consideration, there is only one wave pattern.

Case 3.8 : For $U_{l} \in V$ with $h_{l}>h_{*}$, we have the following asymptotic wave patterns:

Subcase (i) The wave pattern consists of a subsonic constant state $U_{l}$ or a 1-wave in $x<-1$, a stationary wave in $-1 \leq x \leq 1$, and a 2 -wave in $x>1$. The 1-wave, denoted by $\left(U_{l} ; \bar{U}\right), \bar{U} \in R_{1}\left(U_{l}\right) \cup S_{1}\left(U_{l}\right)$, is a 1-shock wave if $\bar{h}>h_{l}$, or a subsonic 1-rarefaction wave if $\tilde{h}_{*}<\bar{h}<h_{l}$, in which $\left(\tilde{h}_{*}, \tilde{u}_{*}\right)=\tilde{U}_{*} \in R_{1}\left(U_{l}\right)$ such that there exists a stationary wave attaining sonic state $\left(\tilde{h}_{0}=g^{-1 / 3}\left(\tilde{h}_{*} \tilde{u}_{*}\right)^{2 / 3}, \tilde{u}_{0}=g^{1 / 3}\left(\tilde{h}_{*} \tilde{u}_{*}\right)^{1 / 3}\right)$ at $x=0$. The stationary wave is continuous and subsonic, and attains the state $\bar{U}$ at $x=1^{-}$. The 2 -wave is a 2 -rarefaction wave or a 2 -shock wave.

Subcase (ii) The wave pattern consists of a subsonic 1-rarefaction wave $\left(U_{l} ; \tilde{U}_{*}\right)$ in $x<$ -1 , a stationary wave with choke in $-1 \leq x \leq 1$, and either a 2 -wave or a 1 -wave followed by a 2 -wave in $x>1$. The stationary wave attains the sonic state at $x=0$, and has one of those Subcases (a)(b)(c) of Subcase (ii)(2) in Case 3.3.

ThEOREM 3.7. Without any assumption of $r$, let $U_{l}=\left(h_{l}, u_{l}\right), h_{*}<h_{l}$, be the given upstream state for the system of shallow water equations (1.1)(1.2). Then there is only one time asymptotic wave pattern for the downstream state $U_{r}$ lying in (see Fig. 15):

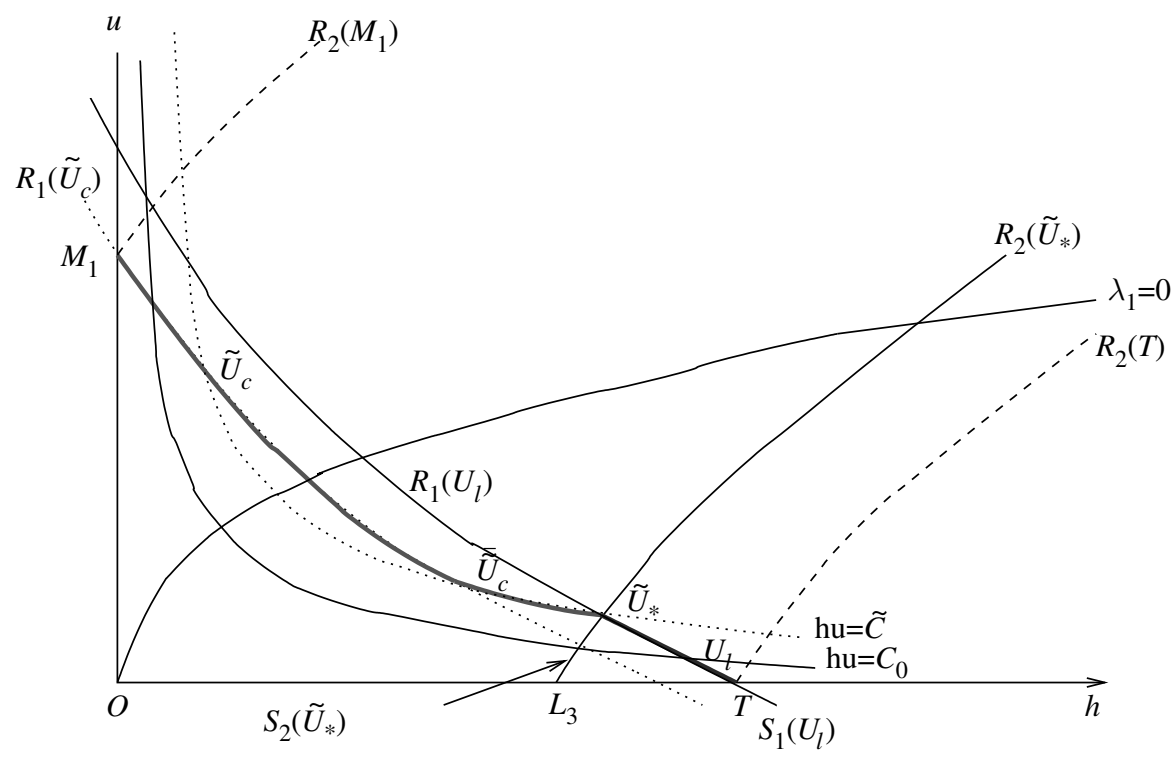

FIG. 15 . 
1. $R_{2}\left(\tilde{U}_{*}\right) \cup\left\{\tilde{U}_{*}\right\} \cup S_{2}\left(\tilde{U}_{*}\right)$ or the open set with boundaries $R_{2}\left(M_{1}\right), \overline{O M_{1}}, \overline{O L_{3}}$, and $R_{2}\left(\tilde{U}_{*}\right) \cup\left\{\tilde{U}_{*}\right\} \cup S_{2}\left(\tilde{U}_{*}\right)$, described as choke + Subcase (ii) of Case 3.8; or

2. the open set with boundaries $R_{2}\left(\tilde{U}_{*}\right) \cup\left\{\tilde{U}_{*}\right\} \cup S_{2}\left(\tilde{U}_{*}\right), \overline{L_{3} T}$, and $R_{2}(T)$, described as Subcase (i) of Case 3.8.

4. Stability. In the previous section, for given upstream state $U_{l}$ and downstream state $U_{r}$, the possible asymptotic solutions are exactly described. When there exists only one solution, it is stable. If not, we want to investigate the stability for the non-unique solutions.

I.1 For fixed $U_{l}, 0<h_{l}<h^{*}$ and $h_{l}$ close to $h_{0}$ :

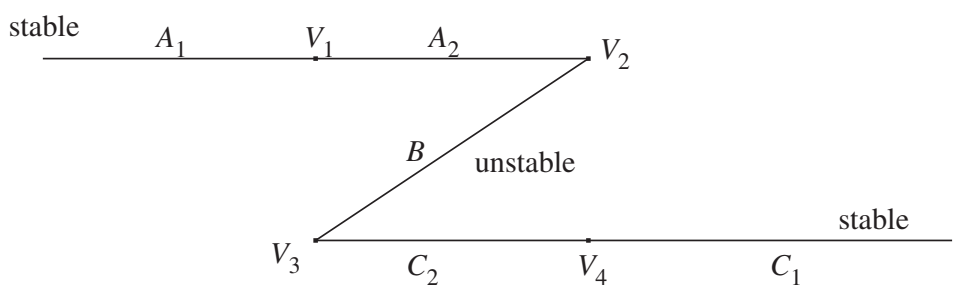

FIG. 16 .

Every point in line $A_{1}$ corresponds to a 2-wave of $U, U \in R_{1}\left(U_{l}\right) \cup\{U \in$ $\left.S_{1}\left(U_{l}\right), \sigma\left(U, U_{l}\right) \geq 0\right\}$ (referring to Fig. 3-1 and Fig. 3-2); every point in $C_{1} \cup$ $\left\{V_{4}\right\} \cup C_{2} \cup\left\{V_{3}\right\}$ corresponds to a 2-wave of $U, U \in S_{1}\left(U_{l}\right), \sigma\left(U, U_{l}\right) \leq 0$ (referring to Fig. 4-1 and Fig. 4-2); points in $\left\{V_{3}\right\} \cup B \cup\left\{V_{2}\right\}$ and $\left\{V_{2}\right\} \cup A_{2} \cup\left\{V_{1}\right\}$ correspond to 2-waves of $U=(h, u), h u=C_{0}, \bar{h}_{l} \leq h \leq h_{Q}$, described in Subcase (iii) and their relating wave patterns have transonic stationary 1 -shocks at $-1 \leq x \leq 0$ and $0 \leq x \leq 1$, respectively (referring to Fig. 5-1 and Fig. 5-2).

The wave pattern of Subcase (i), relating to a point in $A_{1}$, is unique and hence stable. Because $\left\{V_{1}\right\} \cup A_{2}$ can be regarded as originating from $A_{1}$, the wave pattern with transonic stationary 1 -shocks at $0 \leq x \leq 1$ relating to a point in $\left\{V_{1}\right\} \cup A_{2}$ is also stable. Analogously, the wave pattern in Subcase (ii) corresponding to $C_{1}$ is stable, and then the solution relating to $C_{2}$ is also stable. Consequently, the wave pattern with transonic stationary 1-shocks at $-1 \leq x \leq 0$ relating to $B$ is unstable. $V_{2}$ and $V_{3}$ are bifurcation points in Figure 16.

I.2 For fixed $U_{l}, h_{l}=h^{*}$ : Except line $D$, the bifurcation diagram (Fig. 17) is similar

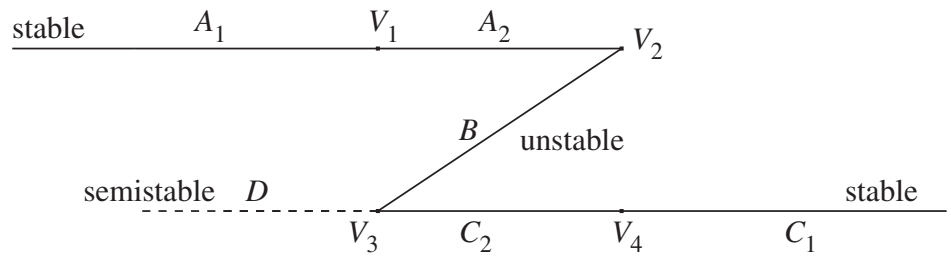

FIG. 17. 
to Fig. 16 and so the stabilities of their relating solutions follow as above. Every point in $D$ corresponds to a 2-wave of $U, U \in R_{1}\left(U_{c}\right) \cup\left\{U \in S_{1}\left(U_{c}\right), \sigma\left(U, U_{c}\right) \geq\right.$ $0\} \cup\left\{U=(h, u): h u=C_{0}, \bar{h}_{c} \leq h \leq h_{*}\right\}$ (the wave pattern contains a choke). The wave patterns relating to $D$ are semistable.

II.1 For fixed $U_{l}, h^{*}<h_{l}<h_{c}$ :

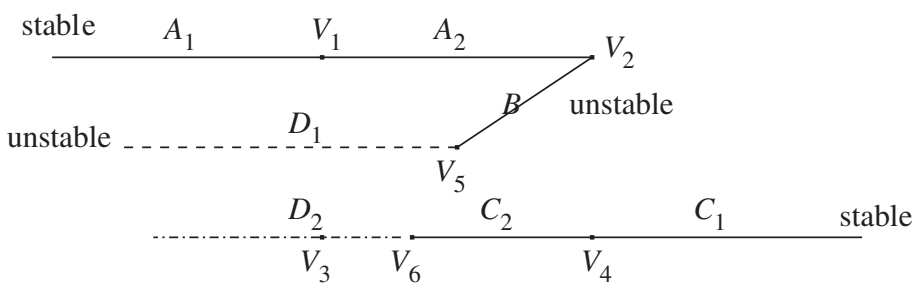

FIG. 18.

Analogously, the phenomenon of chokes happens in these wave patterns relating to $D_{1} \cup\left\{V_{5}\right\}$ and $D_{2} \cup\left\{V_{6}\right\}$. Every point in $D_{1} \cup\left\{V_{5}\right\}$ corresponds to a 2-wave of $U, U \in R_{1}\left(U_{c}\right) \cup\left\{U \in S_{1}\left(U_{c}\right), \sigma\left(U, U_{c}\right) \geq 0\right\} \cup\left\{U=(h, u): h u=C_{0}, \bar{h}_{c} \leq h \leq\right.$ $\left.h_{*}\right\}$ (referring to Fig. 11-2). A point in $D_{2} \cup\left\{V_{6}\right\}$ corresponds to a 2-wave of $U$, $U \in R_{1}\left(\tilde{U}_{c}\right) \cup\left\{U \in S_{1}\left(\tilde{U}_{c}\right), \sigma\left(U, \tilde{U}_{c}\right) \geq 0\right\} \cup\left\{U=(h, u): h u=\tilde{C}, \tilde{\tilde{h}}_{c} \leq h \leq \tilde{h}_{*}\right\}$ (referring to Fig. 10-2).

Since $D_{2}$ originates from $C_{2}$, the relating wave patterns are stable. However, those relating to $D_{1}$ are unstable due to the instability of its origin $B$.

II.2 For fixed $U_{l}, h_{l}=h_{c}$ :

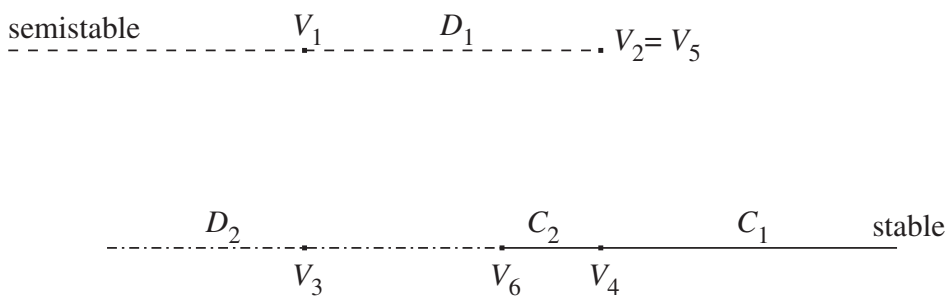

FIG. 19 .

In this case, the wave patterns relating to $C_{1} \cup\left\{V_{4}\right\} \cup C_{2} \cup\left\{V_{6}\right\} \cup D_{2}$ are stable due to the stability of the origin. The wave patterns relating to $\left\{V_{2}\right\} \cup D_{1}$ are semistable.

III For fixed $U_{l}, h_{c}<h_{l}<h_{0}$ :

$\begin{array}{lllll}\mathrm{D}_{2} & \mathrm{C}_{2} & \mathrm{C}_{1} & \text { stable } \\ & \mathrm{V}_{3} & \mathrm{~V}_{6} & \mathrm{~V}_{4} & \end{array}$

FIG. 20 .

The wave pattern is unique and hence stable. 
For $U_{l}$ in regions $I V$ and $V$, the wave pattern is unique and hence stable.

REMARK 4.1. When the supersonic upstream state $h_{l}$ decreases to the critical state $h^{*}$ (from Fig. 18 to Fig. 17), $D_{1}$ (unstable) and $D_{2}$ (stable) in Fig. 18 combine to $D$ in Fig. 17. On the other hand, when $h_{l}$ increases to the critical state $h_{c},\left\{V_{5}\right\} \cup D_{1}$ approaches $\left\{V_{2}\right\} \cup A_{2} \cup\left\{V_{1}\right\} \cup A_{1}$ in Fig. 18. As a result, $D$ in Fig. 17 and $\left\{V_{2}\right\} \cup D_{1} \cup\left\{V_{1}\right\}$ in Fig. 19 are semistable, being the superposition of stable and unstable wave patterns. We therefore conclude that there is transitional semistable asymptotic wave pattern in some region as the upstream state are the critical points $h^{*}$ and $h_{c}$. An unstable pattern would evolve in time to other stable states when the perturbation is not on a manifold in the function space of codimension one. For a semistable pattern there would also exist such a manifold; however, it is nonlinearly stable (or unstable) because the perturbation is from one side (or the other) of the manifold. Our analysis yields this basic information on stability.

It would be interesting to study the issue of stability in time for the wave patterns we have constructed. This is, however, left for the future.

Acknowledgments: We would like to thank Professor T.-P. Liu for thoughtful suggestions and helpful insights. The research is supported by Institute of Mathematics, Academia Sinica and NSC 90-2115-M-001-014, NSC 91007p, and NSC 91008p.

\section{REFERENCES}

[1] F. Bouchut, A. Mangeney-Castelnau, B. Perthame, and J.-P. Vilotte, A new model of Saint Venant and Savage-Hutter type for gravity driven shallow water flows, preprint.

[2] S. Douady, B. Andreotti, and A. Daerr, On granular surface flow equations, Eur. Phys. J. B 11 (1999), 131-142.

[3] J.-F. Gerbeau and B. Perthame, Derivation of viscous Saint-Venant system for laminar shallow water; numerical validation, Discrete and continuous dynamical system- series B 1, no.1 (2001). MR.1821555(2001m:76029)

[4] A. Kurganov and D. Levy, Central-upwind schemes for Saint-Venant system, M2AN Math. Model. Numer. Anal. 36 (2002), no. 3, 397-425. MR1918938 (2003d:76115)

[5] P. D. Lax, Hyperbolic Systems of Conservation Laws and the Mathematical Theory of Shock Waves, (1973). MR0350216 (50:2709)

[6] W.-C. Lien, Hyperbolic Conservation Laws with a moving source, Communication on Pure and Applied Mathematics, Vol. LII, (1999), 1075-1098. MR.1692156|(2000c:35153)

[7] T.-P. Liu, Nonlinear resonance for quasilinear hyperbolic equation, J. Math. Phys. 28 no. 11 (1987), 2593-2602. MR0913412 (88k:35122) 